\title{
ASYMPTOTIC BEHAVIOUR OF THE EMPIRICAL BAYES POSTERIORS ASSOCIATED TO MAXIMUM MARGINAL LIKELIHOOD ESTIMATOR: SUPPLEMENTARY MATERIAL
}

\author{
By Judith Rousseau ${ }^{*, \ddagger, \S}$, Botond Szabo ${ }^{\dagger, \boldsymbol{\Upsilon}, \|}$ \\ University Paris Dauphine $e^{\ddagger}$, and CREST-ENSAE $E^{\S}$, and Budapest \\ University of Technology", and Leiden University" \\ This is the supplementary material associated to the paper Rousseau \\ and Szabo [6]. We provide here the proofs of Propositions 3.1-3.6, to- \\ gether with some technical Lemmas used in the context of priors (T2) \\ and (T3) and some technical Lemmas used in the study of the hierar- \\ chical Bayes posteriors. Finally some Lemmas used in the regression \\ and density estimation problems are given.
}

First of all we note that in the supplementary material we number the Lemmas and displays by letters (e.g. Lemma 2.a or inequality (1.a)) for clarity and better separation from the paper Rousseau and Szabo [6].

\section{Proof of the Propositions.}

1.1. Proof of Proposition 3.1. It is sufficient to prove that all conditions of Theorems 2.1, 2.2, 2.3, and Corollary 2.1 hold, since then the Proposition follows from the combination of them with Lemmas 3.3 and 3.5.

As a first step we note that since there are only finite many truncation parameters $\left(\left|\Lambda_{n}\right|=o(n)\right)$ there is no need to introduce a change of measures $\psi_{k, k^{\prime}}$, one can simply take $q_{k, n}^{\theta}=p_{\theta}^{n}$. Furthermore, we also have from $N_{n}\left(\Lambda_{n}\right)=o(n)$ and $n \varepsilon_{n, 0}^{2} \geq m_{n} \log n$ that $\log N_{n}\left(\Lambda_{n}\right) \lesssim \log n=o\left(n \varepsilon_{n, 0}^{2}\right)$.

Next we define for all $k \leq \varepsilon n / \log n$, with $\varepsilon>0$ fixed but arbitrarily small, the set $\Theta_{n}(k)=\left\{\theta \in \mathbb{R}^{k} ; \max _{j}\left|\theta_{j}\right| \leq\left(M_{n}^{2} n \varepsilon_{n, 0}^{2}\right)^{1 / p^{*}}\right\}$, so that the

\footnotetext{
${ }^{*}$ The project was partially supported by the ANR IPANEMA, the labex ECODEC

${ }^{\dagger}$ The project was partially supported by the labex ECODEC, the European Research Council under ERC Grant Agreement 320637, Netherlands Organization for Scientific Research

AMS 2000 subject classifications: Primary 62G20, 62G05, 60K35; secondary $62 \mathrm{G} 08,62 \mathrm{G} 07$

Keywords and phrases: posterior contraction rates, adaptation, empirical Bayes, hierarchical Bayes, nonparametric regression, density estimation, Gaussian prior, truncation prior
} 
exponential moment condition on $g$ implies that

$$
\Pi\left(\Theta_{n}(k)^{c} \mid k\right) \lesssim k e^{-w_{n}^{2} n \varepsilon_{n, 0}^{2}}, \quad \text { if } w_{n}^{2} \leq s_{0} M_{n}^{2},
$$

and condition (2.6) holds. Furthermore, following from Lemma 3.1 and

$$
\log N\left(\zeta \varepsilon_{n}(k), \Theta_{n}(k),\|\cdot\|_{2}\right) \lesssim k \log n,
$$

for every $\zeta \in(0,1)$, there exists a large enough constant $c(k)=K$ such that the entropy is bounded from above by $c(k)^{2} n \varepsilon_{n}(k)^{2} / 4$. We note that by slicing up the set $\Theta_{n}(k)$, see for instance the proof of Proposition 3.3, the upper bound on the entropy would hold for any $c(k)=K>0$.

From [1] we have that

$$
2 K\left(\theta_{0}, \theta\right)=V_{2}\left(\theta_{0}, \theta\right)=n\left\|f_{\theta_{0}}-f_{\theta}\right\|_{2}^{2}=n\left\|\theta-\theta_{0}\right\|_{2}^{2}
$$

so that (B1) holds with $M_{2}=1$ and $\tilde{\Lambda}_{0}=\left\{k_{n}\right\}$ where $k_{n} \in\left\{\varepsilon_{n}(k) \leq\right.$ $\left.M_{1} \varepsilon_{n, 0}\right\}$. Then conditions (A2), $(C 1)-(C 3)$ follow from [4] with $d_{n}\left(f_{\theta}, f_{\theta_{0}}\right)=$ $\left\|f_{\theta}-f_{\theta_{0}}\right\|_{n}$ the empirical $L_{2}$-distance, which is also equal to the $\ell_{2}$ norm $\left\|\theta-\theta_{0}\right\|_{2}=\left\|f_{\theta}-f_{\theta_{0}}\right\|_{2}$ (from Parseval inequality). Finally condition (2.12) is proved in Lemma 2.b and (H2) in Lemma 4.d.

1.2. Proof of Proposition 3.2. Similarly to Proposition 3.1 it is sufficient to verify that all the conditions of Theorems 2.1, 2.2, 2.3, and Corollary 2.1 hold.

Take $u_{n} \lesssim n^{-3} / \log n$ for $\lambda=\alpha$ and $u_{n} \lesssim n^{-(5 / 2+2 \alpha)}$ for $\lambda=\tau$. Since

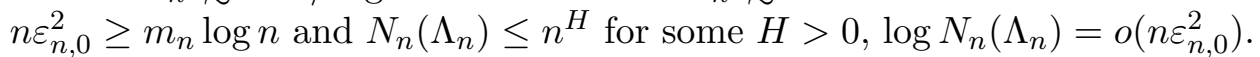
Furthermore condition (B1) follows from Proposition 1 of [1] with $M_{2}=1$.

The proof of conditions (A1) and (A2) are given in Lemma 2.a, Lemma 4.c, and Lemma 4.a with $c_{1}=1 / 2, \zeta=1 / 18, c(\lambda)^{2}=K^{2} \geq 10 \mu / c_{1}$ (where $\mu$ is defined in Lemma 4.a), and $d\left(\theta_{1}, \theta_{2}\right)=\left\|\theta_{1}-\theta_{2}\right\|_{2}$. Condition (H2) holds following from Lemma 4.d with $c_{3}=2+3 \sigma^{-2} K^{2} / 2$. Finally for Corollary 2.1 conditions (C1)-(C2) follow again from the preceding lemmas with $M>$ $10^{3 / 2} \sqrt{\mu}, c_{2}=\mu$, since $w_{n} \varepsilon_{n, 0}=o\left(\varepsilon_{n}(\lambda)\right)$ for all $\lambda \in \Lambda_{n} \backslash \Lambda_{0}$. Note also that from the proof of Lemma 4 .a we also have for $u_{n} \lesssim n^{-2}$ that $\left\|\theta-\psi_{\lambda, \lambda^{\prime}}\right\|_{2}=$ $o\left(n^{-1}\right)=o\left(\varepsilon_{n, 0}\right)$, for every $\left\|\theta-\theta_{0}\right\|=O(1)$.

The lower bound in the case $\alpha+1 / 2 \leq \beta$ follows from Theorem 2.2 and Lemma 3.4, since condition (2.12) is proved in Lemmas 2.b and 4.a.

Finally we note that the same results hold for the Gaussian white noise model as well. The proof can be easily derived from the proof on the regression model, by substituting $e_{j}\left(t_{i}\right)$ by $\delta_{0}(i-j)$ (where $\delta_{0}$ is the Diracdelta measure) in Lemmas 4.c and 4.a and taking $\sigma^{2}=1 / n$ (in this case 
$\left.c_{3}=2+3 K^{2} / 2\right)$. Furthermore one can choose $\zeta=c_{1}=1 / 2$ in the testing assumption (A2) by using the likelihood ratio test in the Gaussian white noise model, see for instance Lemma 5 of [4].

1.3. Proof of Proposition 3.3. The proof consists in showing that assumptions (A1), (A2bis), (B1) and (C1)-(C3) are verified.

In the case of prior (T1), there is no need to consider a change of measure since $\Lambda$ is finite, so that $N_{n}\left(\Lambda_{n}\right)=o(n)$. Then similarly to the proof of Proposition 3.1 we have that $\log N_{n}\left(\Lambda_{n}\right)=o\left(n \varepsilon_{n, 0}^{2}\right)$.

We first prove (B1), or more precisely the variation of (B1) given in Remark 2.2. Choose $k_{0} \in \Lambda_{0}$ which verifies $\varepsilon_{n, 0} \leq \varepsilon_{n}\left(k_{0}\right) \leq M_{1} \varepsilon_{n, 0}$ for some $M_{1} \geq 1$. We have for all $k$ and all $\theta \in R^{k}$ that $\|\theta\|_{1} \leq \sqrt{k}\left\|\theta-\theta_{0}\right\|_{2}+\left\|\theta_{0}\right\|_{1}$. Now let $\theta_{0} \in \mathcal{H}_{\infty}(\beta, L) \cup \mathcal{S}_{\beta}(L)$ with $\beta>1 / 2$, then $\left\|\theta_{0}\right\|_{1}<+\infty$, and if $k_{0} \in \Lambda_{0}$ satisfies $\varepsilon_{n, 0} \leq \varepsilon_{n}\left(k_{0}\right) \leq M_{1} \varepsilon_{n, 0}$ for some $M_{1} \geq 1$, then

$$
\varepsilon_{n}\left(k_{0}\right) \lesssim \frac{\sqrt{k_{0} \log n}}{\sqrt{n}} \vee k_{0}^{-\beta}, \quad \sqrt{k_{0}} \varepsilon_{n}\left(k_{0}\right)=o(1)
$$

so that

$$
\left\{\left\|\theta-\theta_{0}\right\|_{2} \leq K \varepsilon_{n}\left(k_{0}\right)\right\} \subset\left\{\|\theta\|_{1} \leq M\right\},
$$

if $M$ is large enough. Moreover, using Lemma 5.a, for all $M>0$,

$$
\left\{\left\|\theta-\theta_{0}\right\|_{2} \leq K \varepsilon_{n}\left(k_{0}\right)\right\} \cap\left\{\|\theta\|_{1} \leq M\right\} \subset B\left(\theta_{0}, M_{2} \varepsilon_{n}\left(k_{0}\right), 2\right),
$$

and (B1) is verified.

We now verify assumption (A1). We have $q_{k, n}^{\theta}=f_{\theta}^{n}$ for all $\theta \in \mathbb{R}^{k}$, thus (2.5) is obvious and (2.6) follows from [5], (verification of condition $\mathbf{A}$ ), with

$$
\Theta_{n}(k)=\left\{\theta \in \mathbb{R}^{k} ;\|\theta\|_{2} \leq R_{n}(k)\right\}, \quad R_{n}(k)=R_{0}\left(n \varepsilon_{n}(k)^{2}\right)^{1 / p^{*}},
$$

for some $R_{0}>0$ large enough. Similarly the tests in (A2) are the Hellinger tests as in [3] so that (2.7) is satisfied.

We now study the change of distance condition of the version (A2bis) of condition (A2). Define $B_{n, j}(k)=\left\{\theta \in \Theta_{n}(k) ;\left\|\theta-\theta_{0}\right\|_{2} \in\left(j \varepsilon_{n}(k),(j+\right.\right.$ 1) $\left.\left.\varepsilon_{n}(k)\right)\right\}$ for $j \geq K$ and let $\theta \in B_{n, j}(k)$. Since $\left\|\theta_{0}\right\|_{2}<+\infty, B_{n, j}(k) \neq \emptyset$ only if $j \leq 2 R_{n}(k) / \varepsilon_{n}(k)$. Note also that $\sqrt{k} \varepsilon_{n}(k) \lesssim k^{-\beta+1 / 2} \vee k \sqrt{\log n / n} \leq 1$. For all $j \leq j_{0}\left(\sqrt{k} \varepsilon_{n}(k)\right)^{-1}$ with $j_{0}>0$ we have $\left\|\theta-\theta_{0}\right\|_{1} \leq \sqrt{k} \varepsilon_{n}(k)(j+1) \leq$ $j_{0}+1$. Using Lemma 5.a in the Appendix, we obtain that

$$
d\left(f_{0}, f_{\theta}\right) \geq e^{-c_{1}\left(j_{0}+1\right)}\left\|\theta-\theta_{0}\right\|_{2} \geq e^{-c_{1}\left(j_{0}+1\right)} j \varepsilon_{n}(k) .
$$

So that $c(k, j)=e^{-c_{1}\left(j_{0}+1\right)} j$. Moreover using [5], p. 8

$$
d\left(f_{\theta}, f_{\theta^{\prime}}\right) \leq e^{c_{1}\left\|\theta-\theta^{\prime}\right\|_{1}}\left\|\theta-\theta^{\prime}\right\|_{2} \leq e^{c_{1} \sqrt{k}\left\|\theta-\theta^{\prime}\right\|_{2}}\left\|\theta-\theta^{\prime}\right\|_{2},
$$


so that if $\left\|\theta-\theta^{\prime}\right\|_{2} \leq \zeta e^{-2 c_{1}\left(j_{0}+1\right)} j \varepsilon_{n}(k), d\left(f_{\theta}, f_{\theta^{\prime}}\right) \leq \zeta j \varepsilon_{n}(k) e^{-c_{1}\left(j_{0}+1\right)}$ as soon as $k$ or $j_{0}$ is large enough. Thus

$$
\begin{aligned}
\log N\left(\zeta c(k, j) \varepsilon_{n}(k), B_{n, j}(k), d(\cdot, \cdot)\right) & \leq \log N\left(\zeta e^{-2 c_{1}\left(j_{0}+1\right)} j \varepsilon_{n}(k), B_{n, j}(k),\|\cdot\|_{2}\right) \\
& \lesssim k=o\left(n \varepsilon_{n}^{2}(k)\right) .
\end{aligned}
$$

Hence, for $n$ large enough we have for $k \in \Lambda_{n} \backslash \Lambda_{0}$

$$
\sum_{K \leq j \leq j_{0} /\left(\sqrt{k} \varepsilon_{n}(k)\right)} e^{-c_{1} n c(k, j)^{2} \varepsilon_{n}(k)^{2} / 2} \leq e^{-c_{1} e^{-c_{1}\left(j_{0}+1\right)} n \varepsilon_{n}(k)^{2} / 4}=o\left(e^{-n w_{n}^{2} \varepsilon_{n, 0}^{2}}\right),
$$

as soon as $w_{n}=o\left(M_{n}\right)$. Now consider $j>j_{0}\left(\sqrt{k} \varepsilon_{n}(k)\right)^{-1}$ and let $\theta \in$ $B_{n, j}(k)$, from equation (16) in the proof of Lemma 3.1 of [5],

$$
d\left(f_{0}, f_{\theta}\right) \gtrsim\left\|\theta-\theta_{0}\right\|_{2}\left(\sqrt{k} \varepsilon_{n}(k) j+\left|\log \left(j \varepsilon_{n}(k)\right)\right|\right)^{-1} .
$$

For all $j \gtrsim \log (k) /\left(\sqrt{k} \varepsilon_{n}(k)\right)$ we have $\sqrt{k} \varepsilon_{n}(k) j \gtrsim\left|\log \left(j \varepsilon_{n}(k)\right)\right|$ and

$$
d\left(f_{0}, f_{\theta}\right) \gtrsim \frac{1}{\sqrt{k}}
$$

when $n$ is large enough and we can choose $c(k, j)=c k^{-1 / 2} \varepsilon_{n}(k)^{-1}$. For all $\theta, \theta^{\prime} \in B_{n, j}(k)$, using equation (8) of [5]

$$
d\left(f_{\theta}, f_{\theta^{\prime}}\right) \lesssim \sqrt{k}\left\|\theta-\theta^{\prime}\right\|_{2},
$$

so that there exists $c>0$

$$
\begin{aligned}
\log N\left(\zeta \frac{\varepsilon_{n}(k)}{\sqrt{k}}, B_{n, j}(k), d(\cdot, \cdot)\right) & \leq \log N\left(\zeta \frac{\varepsilon_{n}(k) c}{k}, B_{n, j}(k),\|\cdot\|_{2}\right) \\
& \lesssim k \log (j k)=o(n / k)
\end{aligned}
$$

for all $j \leq R_{n}(k) / \varepsilon_{n}(k)$ and for all $C_{1}, C_{2}>0$

$$
\sum_{j=\left\lceil C_{1} \log k /\left(\sqrt{k} \varepsilon_{n}(k)\right)\right\rceil}^{\left\lfloor R_{n}(k) / \varepsilon_{n}(k)\right\rfloor} e^{-C_{2} n / k} \leq e^{-C_{2} n / k} \frac{R_{n}(k)}{\varepsilon_{n}(k)} \leq e^{-\frac{C_{2} n}{2 k}}, \quad n \text { large enough. }
$$

Combining (1.c) with

$$
n^{1 /(2 \beta+1)}(\log n)^{2 \beta /(2 \beta+1)} \gtrsim n \varepsilon_{n, 0}^{2}, \quad \text { and } \quad \frac{n}{k} \geq \sqrt{n}, \quad \forall k \lesssim \sqrt{n},
$$


implies that

$$
\sum_{j=\left\lceil C_{1} \log k /\left(\sqrt{k} \varepsilon_{n}(k)\right)\right\rceil}^{\left\lfloor R_{n}(k) / \varepsilon_{n}(k)\right\rfloor} e^{-C_{2} n / k}=o\left(e^{-n w_{n}^{2} \varepsilon_{n, 0}^{2}}\right),
$$

when $w_{n}^{2}=o\left(n^{(\beta-1 / 2-\delta) /(2 \beta+1)}\right)$ with $0<\delta<\beta-1 / 2$.

We now consider $j_{0} /\left\{\sqrt{k} \varepsilon_{n}(k)\right\} \leq j \leq \delta \log (k) /\left\{\sqrt{k} \varepsilon_{n}(k)\right\}$ with $\delta$ arbitrarily small. Then

$$
d\left(f_{0}, f_{\theta}\right) \gtrsim\left\|\theta-\theta_{0}\right\|_{2}\left(\left|\log \left(j \varepsilon_{n}(k)\right)\right|\right)^{-1} \gtrsim \frac{j \varepsilon_{n}(k)}{\log n}
$$

so that $c(k, j) \gtrsim j / \log n$. Note also that, similarly to before, this implies that $d\left(f_{0}, f_{\theta}\right)>\delta_{n} \varepsilon_{n, 0}$ as soon as $k \leq k_{0}(n / \log n)^{1 /(2 \beta+1)}$ for all $k_{0}>0$ and $n$ large enough. Using (1.b), $\log N\left(\zeta \frac{\varepsilon_{n}(k)}{\sqrt{k}}, B_{n, j}(k), d(\cdot, \cdot)\right) \lesssim k \log (k)$. Moreover

$$
n c(k, j)^{2} \varepsilon_{n}(k)^{2} \gtrsim \frac{n \varepsilon_{n}(k)^{2} j^{2}}{(\log n)^{2}} \gtrsim \frac{n j_{0}^{2}}{(\log n)^{2} k}
$$

for all $j \geq j_{0} /\left(\sqrt{k} \varepsilon_{n}(k)\right)$. By choosing $j_{0}$ large enough, we thus have that for $k_{0}$ fixed and all $k \leq k_{0} \sqrt{n}(\log n)^{-3}$,

$$
\log N\left(\zeta \frac{\varepsilon_{n}(k)}{\sqrt{k}}, B_{n, j}(k), d(\cdot, \cdot)\right) \leq c_{1} n c(k, j)^{2} \varepsilon_{n}(k)^{2} / 2 .
$$

We also have that

$$
\sum_{j=\left\lceil j_{0}\left(\sqrt{k} \varepsilon_{n}(k)\right)^{-1}\right\rceil}^{\left\lceil C_{1} \log k /\left(\sqrt{k} \varepsilon_{n}(k)\right)\right\rceil+1} e^{-\frac{C_{2} n}{2 k}} \leq o\left(e^{-n w_{n}^{2} \varepsilon_{n, 0}^{2}}\right) .
$$

Combining (1.a), (1.d) and (1.e), we finally prove (A2bis).

We now verify conditions $(\mathrm{C} 1)-(\mathrm{C} 3)$ to obtain the posterior concentration rate. We already know from Lemma 3.1 that $\varepsilon_{n, 0} \lesssim(n / \log n)^{-\beta /(2 \beta+1)}$ where the constant depends only on $L, \beta_{1}$, and $\beta_{2}$ if $\theta_{0} \in \mathcal{S}_{\beta}(L)$. Since we do not need the change of measures $\psi_{\lambda, \lambda^{\prime}},(\mathrm{C} 1)$ and $(\mathrm{C} 2)$ are proved in [5].

Finally for the lower bound on the contraction rate condition (2.12), take $\theta_{0 i}^{2}=i^{-2 \beta-1} \in \mathcal{H}(\beta, L)$, so that $\varepsilon_{n, 0} \asymp(n / \log n)^{-\beta /(2 \beta+1)}$ and if $\left\|\theta-\theta_{0}\right\|_{2} \leq$ $M \delta \varepsilon_{n, 0}$ with $\theta \in \mathbb{R}^{k}$ and $M>0$ then $k \gtrsim \delta_{n}^{-1 / \beta}(n / \log n)^{1 /(2 \beta+1)}$. The above computations imply also that if there exists $k \lesssim \delta_{n}^{-1 / \beta}(n / \log n)^{1 /(2 \beta+1)}$ then $d\left(f_{0}, f_{\theta}\right) \gtrsim\left\|\theta-\theta_{0}\right\|_{2}$ on the set $\left\{d\left(f_{0}, f_{\theta}\right) \leq \delta_{n} \varepsilon_{n, 0}\right\}$ so that Lemma 3.1 implies condition (2.12).

For the hierarchical Bayes result assumption (H2) is verified in Lemma 5.c. 
1.4. Proof of Proposition 3.4. To prove Proposition 3.4 we need to verify that (A1)-(A2) and (B1) are satisfied, together with (C1)-(C3), (2.12) and (H2). Let $\tau_{0} \in \Lambda_{0}$ satisfying $M_{1} \varepsilon_{n, 0} \geq \varepsilon_{n}\left(\tau_{0}\right)$. Equation (5.d) in Lemma 5.b, with $K_{n}=\left\lfloor\left(\tau_{0}^{2} n \varepsilon_{n, 0}^{2}\right)^{1 /(2 \alpha)}\right\rfloor$ implies that for $M \geq\left\|\theta_{0}\right\|_{1}$,

$\Pi\left(\left\|\theta-\theta_{0}\right\|_{2} \leq K \varepsilon_{n}\left(\tau_{0}\right) ;\left\|\theta-\theta_{0}\right\|_{1} \leq K \sqrt{K_{n}} \varepsilon_{n}\left(\tau_{0}\right)+2 M \mid \alpha, \tau_{0}\right) \gtrsim e^{-n M^{2} \varepsilon_{n, 0}^{2} / 2}$.

Moreover $\sqrt{K_{n}} \varepsilon_{n}\left(\tau_{0}\right) \lesssim\left(\tau_{0}^{2} n \varepsilon_{n, 0}^{2}\right)^{1 /(4 \alpha)} \varepsilon_{n}\left(\tau_{0}\right)$ and using Lemmas 3.2 and 3.3 if $\beta \leq \alpha+1 / 2$,

$$
\begin{aligned}
\sqrt{K_{n}} \varepsilon_{n}\left(\tau_{0}\right) & \lesssim n^{1 /[(2 \beta+1) 4 \alpha]-\beta /(2 \beta+1)} \tau_{0}^{1 /(2 \alpha)}, \quad \tau_{0} \lesssim n^{-\frac{\beta(2 \alpha+1)}{2 \beta+1}+\alpha} \\
\sqrt{K_{n}} \varepsilon_{n}\left(\tau_{0}\right) & \lesssim n^{1 /[(2 \beta+1) 4 \alpha]-\beta /(2 \beta+1)+1 / 2-\beta(2 \alpha+1) /[2 \alpha(2 \beta+1)]} \\
& =n^{-\frac{(2 \beta-1)(2 \alpha+1)}{4 \alpha(2 \beta+1)}}=o(1) .
\end{aligned}
$$

Similarly if $\beta>\alpha+1 / 2$,

$$
\begin{aligned}
& \sqrt{K_{n}} \varepsilon_{n}\left(\tau_{0}\right) \lesssim n^{1 /[(\alpha+1) 8 \alpha]-(2 \alpha+1) /(4 \alpha+4)} \tau_{0}^{1 /(2 \alpha)}, \quad \tau_{0} \lesssim n^{-\frac{(2 \alpha+1)^{2}}{4 \alpha+4}+\alpha}, \\
& \sqrt{K_{n}} \varepsilon_{n}\left(\tau_{0}\right) \lesssim n^{-\frac{2 \alpha+1}{4(\alpha+1)}}=o(1) .
\end{aligned}
$$

So that for $n$ large enough,

$$
\Pi\left(\left\|\theta-\theta_{0}\right\|_{2} \leq K \varepsilon_{n}\left(\tau_{0}\right) ;\left\|\theta-\theta_{0}\right\|_{1} \leq 3 M \mid \alpha, \tau_{0}\right) \gtrsim e^{-n M^{2} \varepsilon_{n, 0}^{2} / 2}
$$

and using the same computations as in the verification of (B1) in Section 1.3 we obtain

$$
\left\{\left\|\theta-\theta_{0}\right\|_{2} \leq K \varepsilon_{n}\left(\tau_{0}\right)\right\} \cap\left\{\|\theta\|_{1} \leq M\right\} \subset B\left(\theta_{0}, M_{2} \varepsilon_{n}\left(\tau_{0}\right), 2\right) .
$$

We now verify (A1), (A2), (C1)-(C3). Consider the transformation defined in (3.11). Then

$$
\begin{aligned}
\log f_{\psi_{\tau, \tau^{\prime}}(\theta)} & =\frac{\tau^{\prime}}{\tau}\left(\sum_{i} \theta_{i} \varphi_{i}\right)-\log \left(\int_{0}^{1} e^{\tau^{\prime} / \tau \sum_{i} \theta_{i} \varphi_{i}(x)} d x\right) \\
& \leq \frac{\tau^{\prime}}{\tau}\left(\sum_{i} \theta_{i} \varphi_{i}-c(\theta)\right)
\end{aligned}
$$

if $\tau^{\prime} \geq \tau$.

$$
\Theta_{n}(\tau)=\left\{\theta=\varepsilon_{n}(\tau) \theta_{1}+R_{n}(\tau) \theta_{2}, \quad \theta_{1} \in \mathbb{B}_{1}, \theta_{2} \in \mathbb{H}_{1}^{\tau}\right\} \cap\left\{\left\|\theta_{1}\right\|_{1} \leq \sqrt{n}\right\},
$$


with $R_{n}(\tau), \mathbb{B}_{1}, \mathbb{H}_{1}^{\tau}$ defined in Lemma 2.a. Lemma 2.a implies that

$$
\Pi\left(\Theta_{n}(\tau)^{c} \mid \alpha, \tau\right) \leq e^{-c n \varepsilon_{n}^{2}(\tau)} .
$$

For all $\theta \in \Theta_{n}(\tau)$,

$\|\theta\|_{1} \leq \varepsilon_{n}(\tau)\left\|\theta_{1}\right\|_{1}+R_{n}(\tau)\left\|\theta_{2}\right\|_{1} \leq \sqrt{n} \varepsilon_{n}(\tau)+R_{n}(\tau) \tau\left(\left\|\theta_{2}\right\|_{\mathbb{H} \tau}+\alpha^{-1}\right) \leq C(\tau) \sqrt{n} \varepsilon_{n}(\tau)$,

so that if $\tau \leq \tau^{\prime} \leq \tau\left(1+u_{n}\right)$ with $u_{n}=o\left(n^{-3 / 2} \varepsilon_{n}(\tau)^{-1}\right)$

$$
q_{\tau, n}^{\theta}\left(\mathbf{x}_{n}\right) \leq f_{\theta}^{n}\left(\mathbf{x}_{n}\right) e^{2 n u_{n}\left\|\sum_{i} \theta_{i} \varphi\right\|_{\infty}} \leq(1+o(1)) f_{\theta}^{n}\left(\mathbf{x}_{n}\right)
$$

and $Q_{n, \tau}^{\theta}\left(\mathcal{X}^{n}\right) \leq 2$ for $n$ large enough, and condition (2.5) in (A1) is satisfied with $\tau_{i}=\underline{\tau}_{n}\left(1+u_{n}\right)^{i}$ the smallest point in the $(i+1) \operatorname{th} \operatorname{bin}\left[\underline{\tau}_{n}\left(1+u_{n}\right)^{i}, \underline{\tau}_{n}(1+\right.$ $\left.u_{n}\right)^{i+1}$ ] on $\Lambda_{n}$. Using (1.h), we also have that

$$
\begin{aligned}
\int_{\Theta_{n}(\tau)^{c}} & q_{\tau, n}^{\theta}\left(\mathcal{X}^{n}\right) d \Pi(\theta \mid \tau) \leq \int_{\Theta_{n}(\tau)^{c}} e^{2 n u_{n}\left\|\sum_{i} \theta_{i} \varphi\right\|_{\infty}} d \Pi(\theta \mid \tau) \\
& \leq \Pi\left(\Theta_{n}(\tau)^{c} \mid \alpha, \tau\right)^{1 / 2}\left(\int e^{2 n u_{n}\left\|\sum_{i} \theta_{i} \varphi\right\|_{\infty}} d \Pi(\theta \mid \tau)\right)^{1 / 2} \\
& \leq e^{-c n \varepsilon_{n}^{2}(\tau) / 2} \prod_{i} 2 e^{n^{2} u_{n}^{2}\|\varphi\|_{\infty}^{2} \tau^{2} i^{-2 \alpha-1}} \\
& \leq e^{2 n^{2} u_{n}^{2}\|\varphi\|_{\infty}^{2} \tau^{2} \sum_{i} i^{-2 \alpha-1}} e^{-c n \varepsilon_{n}^{2}(\tau) / 2} \leq 2 e^{-c n \varepsilon_{n}^{2}(\tau) / 2},
\end{aligned}
$$

if $u_{n}=o\left(n^{-1} \tau^{-1}\right)$, and condition (2.6) is verified.

Similarly to the case of prior (T1) the tests in condition (A2) are the Hellinger tests as in [3] so that (2.7) is satisfied using (1.h). We now verify (2.8). Recall that for all $\theta_{0} \in \mathcal{S}_{\beta}(L) \cup \mathcal{H}_{\infty}(\beta, L)$ with $L>0$ and $\beta>1 / 2$, $\left\|\theta_{0}\right\|_{1}<+\infty$. From Lemma 5.a,

$$
d\left(f_{0}, f_{\theta}\right) \gtrsim\left\|\theta-\theta_{0}\right\|_{2} e^{-C\left\|\theta-\theta_{0}\right\|_{1}} .
$$

Define $\tilde{\Theta}_{n}(\tau)=\Theta_{n}(\tau) \cap\left\{\left\|\theta-\theta_{0}\right\|_{2}>K \varepsilon_{n}(\tau) ;\left\|\theta-\theta_{0}\right\|_{1} \leq \sqrt{K_{n}}\left\|\theta-\theta_{0}\right\|_{2}+\right.$ $M\} \cap \bar{\Theta}_{n}(\tau)$ with $K_{n}^{2 \alpha}=n \tau^{2} w_{n}^{2} \varepsilon_{n, 0}^{2}, M \geq 2\left\|\theta_{0}\right\|_{1}$ and

$$
\bar{\Theta}_{n}(\tau)=u_{n} \mathbb{B}_{1}^{L_{1}}+\bar{R}_{n} \mathbb{H}_{1}
$$

with $u_{n}=\tau C_{\alpha}^{-(\alpha-1 / 2)}\left(n \varepsilon_{n, 0}^{2} w_{n}\right)^{-(\alpha-1 / 2)} \wedge M$ and $\mathbb{B}_{1}^{L_{1}}$ is the $L_{1}$ unit ball and $\bar{R}_{n} \asymp n \varepsilon_{n, 0}^{2} w_{n}^{1 / 2}$ if $\tau C_{\alpha}^{-(\alpha-1 / 2)}\left(n \varepsilon_{n, 0}^{2} w_{n}\right)^{-(\alpha-1 / 2)} \leq M$ else $\bar{R}_{n} \asymp C_{\alpha}^{1 / 2}\left(\frac{M}{\tau}\right)^{-\frac{1}{2(\alpha-1 / 2)}}$. From (1.g), (2.b) and Lemma 5.b

$$
\Pi\left(\tilde{\Theta}_{n}^{c} \mid \tau\right) \leq e^{-c n \varepsilon_{n}(\tau)^{2}}+e^{-A n w_{n} \varepsilon_{n, 0}^{2}}
$$


where $A$ can be chosen as large as need be by choosing $C_{\alpha}$ large enough. If $\theta \in \tilde{\Theta}_{n}(\tau)$ with $\left\|\theta-\theta_{0}\right\|_{2} \leq M K_{n}^{-1 / 2}$ then from Lemma 5.a $d\left(f_{0}, f_{\theta}\right) \gtrsim$ $\left\|\theta-\theta_{0}\right\|_{2} e^{-2 C M}$; so that $c(\tau) \geq e^{-2 C M}$. Now let $\left\|\theta-\theta_{0}\right\|_{2}>M K_{n}^{-1 / 2}$. Note that, from Lemma 3.2,

$$
\varepsilon_{n}(\tau) \leq n^{-\alpha /(2 \alpha+1)} \tau^{1 /(2 \alpha+1)}+\left(\frac{C}{n \tau^{2}}\right)^{\frac{\beta}{2 \alpha+1} \wedge \frac{1}{2}}
$$

and $\varepsilon_{n, 0} \leq n^{-(2 \alpha+1) /(4 \alpha+4)}$ if $\beta>\alpha+1 / 2$ and else $\varepsilon_{n, 0} \leq n^{-\beta /(2 \beta+1)}$. This implies that for all $\tau \leq \bar{\tau}_{n}=n^{\alpha / 2-1 / 4}$

$$
\varepsilon_{n}(\tau)\left(n \tau^{2} w_{n}^{2} \varepsilon_{n, 0}^{2}\right)^{1 /(4 \alpha)}<M(\log n)^{-2},
$$

which combined with $\left\|\theta-\theta_{0}\right\|_{2}>M K_{n}^{-1 / 2}$, leads to $\left\|\theta-\theta_{0}\right\|_{2}>(\log n)^{2} \varepsilon_{n}(\tau)$. Theorem 5.1 of [8] implies that either $d\left(f_{0}, f_{\theta}\right) \geq 1-1 / e$ or

$$
V_{2}\left(f_{0}, f_{\theta}\right) \leq C d^{2}\left(f_{0}, f_{\theta}\right)\left(1+(\log n)^{2}+\|\theta\|_{1}^{2}\right) .
$$

Moreover, since $f_{0} \geq c_{0}$,

$$
\begin{aligned}
V_{2}\left(f_{0}, f_{\theta}\right) & \geq c_{0} \int_{0}^{1}\left(\sum_{j \geq 2}\left(\theta_{j}-\theta_{0, j}\right)\left(\varphi_{j}-c_{j}\left(f_{0}\right)\right)\right)^{2} d x \\
& =c_{0}\left(\left\|\theta-\theta_{0}\right\|_{2}+\left(\sum_{j}\left(\theta_{j}-\theta_{0, j}\right) c_{j}\left(f_{0}\right)\right)^{2}\right) \geq c_{0}\left\|\theta-\theta_{0}\right\|_{2}^{2}
\end{aligned}
$$

and

$$
d\left(f_{0}, f_{\theta}\right) \gtrsim \frac{\left\|\theta-\theta_{0}\right\|_{2}}{1+\log n+\left\|\theta-\theta_{0}\right\|_{2} \sqrt{K_{n}}} \gtrsim K_{n}^{-1 / 2} / \log n
$$

so that $d\left(f_{0}, f_{\theta}\right) \gtrsim \varepsilon_{n}(\tau) \log n$ and (2.8) is verified with $c(\tau)>0$. To verify condition (2.9), we need to control the Hellinger entropy. Over the subset of $\tilde{\Theta}_{n}(\alpha, \tau)$ defined by $\left\|\theta-\theta_{0}\right\|_{2} \leq M / \sqrt{K_{n}},\left\|\theta-\theta_{0}\right\|_{1} \leq M$ and lemmas 5.a and 2.a imply that the Hellinger entropy of this set is bounded by the $L_{2}$ entropy which is bounded by $C n \varepsilon_{n}^{2}(\alpha, \tau)$. If $\left\|\theta-\theta_{0}\right\|_{2}>M / \sqrt{K_{n}}$, the above computations imply that $d\left(f_{0}, f_{\theta}\right) \gtrsim M K_{n}^{-1 / 2}(\log n)^{-1}$. Moreover, if $\theta \in \tilde{\Theta}_{n}(\alpha, \tau)$ and $\left\|\theta-\theta^{\prime}\right\|_{2} \leq \varepsilon_{n}(\alpha, \tau)$, then for all $J>0$

$$
\sum_{j \leq J}\left|\theta_{j}-\theta_{j}^{\prime}\right| \leq \sqrt{J}\left\|\theta-\theta^{\prime}\right\|_{2} \leq \sqrt{J} \varepsilon_{n}(\alpha, \tau) .
$$


Choose $J \asymp \varepsilon_{n}(\alpha, \tau)^{-2}$. Then, since $\theta=\theta_{1}+\theta_{2}$ and $\theta^{\prime}=\theta_{1}^{\prime}+\theta_{2}^{\prime}$ with $\theta_{1}, \theta_{1}^{\prime} \in u_{n} \mathbb{B}_{1}^{L_{1}}$ and $\theta_{2}, \theta_{2}^{\prime} \in \bar{R}_{n} \mathbb{H}_{1}$,

$$
\sum_{j \geq J+1}\left|\theta_{j}-\theta_{j}^{\prime}\right| \leq 2 u_{n}+2 \alpha^{-1 / 2} \bar{R}_{n} J^{-\alpha} \lesssim u_{n}+\sqrt{\varepsilon_{n}(\alpha, \tau)^{4 \alpha} n \varepsilon_{n, 0}^{2} w_{n}},
$$

if $\tau C_{\alpha}^{-(\alpha-1 / 2)}\left(n \varepsilon_{n, 0}^{2} w_{n}\right)^{-(\alpha-1 / 2)} \leq M$ or

$$
\sum_{j \geq J+1}\left|\theta_{j}-\theta_{j}^{\prime}\right| \lesssim u_{n}+\sqrt{\varepsilon_{n}(\alpha, \tau)^{4 \alpha} \tau^{1 /(\alpha-1 / 2)}},
$$

if $\tau C_{\alpha}^{-(\alpha-1 / 2)}\left(n \varepsilon_{n, 0}^{2} w_{n}\right)^{-(\alpha-1 / 2)}>M$. So that $\left\|\theta-\theta^{\prime}\right\|_{1}=O(1)$ as soon as $\varepsilon_{n}(\alpha, \tau)^{4 \alpha} n \varepsilon_{n, 0}^{2} w_{n}=O(1)$. In the case $\beta \leq \alpha+1 / 2$ and $\tau=o\left(n^{(\alpha-1 / 2) /(2 \beta+1)}\right)$, $(1.1)$

$n^{-4 \alpha^{2} /(2 \alpha+1)} \tau^{4 \alpha /(2 \alpha+1)}=o\left(n^{-1 /(2 \beta+1)}\right), \quad\left(n \tau^{2}\right)^{-4 \alpha \beta /(2 \alpha+1)}=o\left(n^{-1 /(2 \beta+1)}\right)$,

the former relation is satisfied when $\tau \lesssim n^{\alpha / 2-1 / 4}$ while the latter requires $\tau \gg n^{-1 / 4+1 /(8 \alpha)}$. In the case $\tau \gtrsim n^{(\alpha-1 / 2) /(2 \beta+1)}$, (1.l) is replaced with $n^{-4 \alpha^{2} /(2 \alpha+1)} \tau^{4 \alpha /(2 \alpha+1)} \tau^{1 /(\alpha-1 / 2)}=o(1)$, which is satisfied as soon as $\tau \leq$ $n^{\alpha / 2-1 / 4}$. In the case $\beta>\alpha+1 / 2$ the same results hold.

Conditions $(\mathrm{C} 1)-(\mathrm{C} 3)$ are direct consequences of the transformation (3.11) which in turns implies (1.h), combined with the definition of $\Theta_{n}(\tau)$ so that (C1) and (C2) hold.

Finally $N_{n}\left(\Lambda_{n}\right)$ is at most polynomial in $n$ so that $\log N_{n}\left(\Lambda_{n}\right)=o\left(n \varepsilon_{n, 0}^{2}\right)$. This terminates the proof of the upper bound on the contraction rate of the MMLE empirical Bayes posterior. Then the lower bound in case $\beta>\alpha+1 / 2$ and $\|\theta\|_{2}>c>0$ follows from the combination of Theorem 2.2 and Lemmas 2.b and 3.3 together with the fact that when $\theta \in \tilde{\Theta}_{n}(\tau)$, either $d\left(f_{0}, f_{\theta}\right) \gtrsim$ $\left\|\theta-\theta_{0}\right\|_{2}$ or $d\left(f_{0}, f_{\theta}\right) \gtrsim K_{n}^{-1 / 2} \asymp n^{-1 /(4 \alpha(\alpha+1))} \tau^{-1 / \alpha} \gtrsim \varepsilon_{n, 0}$.

Finally for the hierarchical Bayes result we note that the transformation (3.11) implies, as in (1.h) that

$$
\ell_{n}\left(\psi_{\tau, \tau^{\prime}}(\theta)\right) \geq-2 n u_{n}\|\varphi\|_{\infty} \sum_{i}\left|\theta_{i}\right|+\ell_{n}(\theta), \quad \text { if } \tau^{\prime} \leq \tau,
$$

which combined with Lemma 5.c and the definition of $\varepsilon_{n, 0}$ implies (H2) as soon as $M_{2} n \varepsilon_{n, 0}^{2} \geq M_{0} \log n$ with $M_{0}$ large enough and $u_{n} \lesssim 1 / n$. Then the statement is a direct consequence of Theorem 2.3.

1.5. Proof of Proposition 3.5. As in the proof of Proposition 3.4, Let $\alpha_{0}$ replacing $\tau_{0}$ in the verification of (B1) in Section 1.4. Equation (5.d) in 
Lemma 5.b, with $K_{n}=\left\lfloor\left(n \varepsilon_{n, 0}^{2}\right)^{1 /\left(2 \alpha_{0}\right)}\right\rfloor$ implies that for $M>\left\|\theta_{0}\right\|_{1}$,

$\Pi\left(\left\|\theta-\theta_{0}\right\|_{2} \leq K \varepsilon_{n}\left(\alpha_{0}\right) ;\left\|\theta-\theta_{0}\right\|_{1} \leq K \sqrt{K_{n}} \varepsilon_{n}\left(\alpha_{0}\right)+2 M \mid \alpha_{0}\right) \gtrsim e^{-\frac{M^{2} n \varepsilon_{n, 0}^{2}}{2}}$

Moreover $\sqrt{K_{n}} \varepsilon_{n}\left(\alpha_{0}\right) \lesssim\left(n \varepsilon_{n, 0}^{2}\right)^{1 /\left(4 \alpha_{0}\right)} \varepsilon_{n}\left(\alpha_{0}\right)$ and by using Lemmas 3.2 and 3.3. (i.e. $n^{-\alpha_{0} /\left(2 \alpha_{0}+1\right)} \lesssim \varepsilon_{n}\left(\alpha_{0}\right) \lesssim(n / \log n)^{-\beta /(2 \beta+1)}, \alpha_{0} \geq \beta$ ) we have that

$$
\begin{array}{r}
\sqrt{K_{n}} \varepsilon_{n}\left(\tau_{0}\right) \lesssim(n / \log n)^{1 /\left[(2 \beta+1) 4 \alpha_{0}\right]-\beta /(2 \beta+1)}=o(1) \quad \text { and } \\
\Pi\left(\left\|\theta-\theta_{0}\right\|_{2} \leq K \varepsilon_{n}\left(\alpha_{0}\right) ;\left\|\theta-\theta_{0}\right\|_{1} \leq 3 M \mid \alpha_{0}\right) \gtrsim e^{-M^{2} n \varepsilon_{n, 0}^{2} / 2} .
\end{array}
$$

As in the case of type (T2) prior,

$$
\left\{\left\|\theta-\theta_{0}\right\|_{2} \leq K \varepsilon_{n}\left(\alpha_{0}\right) ;\left\|\theta-\theta_{0}\right\|_{1} \leq 3 M\right\} \subset B\left(\theta_{0}, M_{2} \varepsilon_{n}\left(\alpha_{0}\right), 2\right),
$$

for some constant $M_{2}>0$.

To study conditions (A1), (A2) and (C1)-(C3), recall that the change of variable $\psi_{\alpha, \alpha^{\prime}}(\theta)$ is defined by (3.12) so that when $\alpha^{\prime} \geq \alpha$

$$
\begin{aligned}
\log f_{\psi_{\alpha, \alpha^{\prime}}(\theta)}(x)-\log f_{\theta}(x)= & \sum_{i}\left(i^{\alpha-\alpha^{\prime}}-1\right) \theta_{i} \varphi_{i} \\
& -\log \left\{\int_{0}^{1} f_{\theta}(x) \exp \left(\sum_{i}\left(i^{\alpha-\alpha^{\prime}}-1\right) \theta_{i} \varphi_{i}(x)\right) d x\right\} \\
\leq & 2\left|\alpha-\alpha^{\prime}\right|\|\theta\|_{1}\|\varphi\|_{\infty} .
\end{aligned}
$$

Let $\alpha \in \Lambda_{n} \backslash \Lambda_{0}$ and define

$$
\Theta_{n}(\alpha)=\left\{\theta=A \varepsilon_{n}(\alpha) \theta_{1}+R_{n}(\alpha) \theta_{2}, \quad \theta_{1} \in \mathbb{B}_{1}, \theta_{2} \in \mathbb{H}_{1}^{\alpha}\right\} \cap\left\{\left\|\theta_{1}\right\|_{1} \leq \sqrt{n}\right\} .
$$

For all $\theta \in \Theta_{n}(\alpha)$,

$\|\theta\|_{1} \leq \varepsilon_{n}(\alpha)\left\|\theta_{1}\right\|_{1}+R_{n}(\alpha)\left\|\theta_{2}\right\|_{1} \leq \sqrt{n} \varepsilon_{n}(\alpha)+R_{n}(\alpha)\left\|\theta_{2}\right\|_{\mathbb{H}^{\alpha}} \alpha^{-1} \leq C \sqrt{n} \varepsilon_{n}(\alpha)$,

for some constant $C$ independent of $\alpha$. Let $\theta \in\left\{\left\|\theta-\theta_{0}\right\|_{2} \leq K \varepsilon_{n}(\alpha)\right\} \cap \Theta_{n}(\alpha)$.

Then for all $\alpha \leq \alpha^{\prime} \leq \alpha+u_{n}$

$$
q_{\alpha, n}^{\theta}\left(\mathbf{x}_{n}\right) \leq e^{2 C n^{3 / 2} \varepsilon_{n}(\alpha)\|\varphi\|_{\infty} u_{n}} f_{\theta}^{n}\left(\mathbf{x}_{n}\right)
$$

so that (2.5) is verified on $\left\{\left\|\theta-\theta_{0}\right\|_{2} \leq K \varepsilon_{n}(\alpha)\right\} \cap \Theta_{n}(\alpha)$ as soon as $u_{n} \leq$ $a n^{-3 / 2} \varepsilon_{n}(\alpha)^{-1}, \forall a>0$ when $n$ is large enough. To prove (2.6), we decompose $\Theta_{n}(\alpha)^{c}$ into $\Theta_{n, j}=\Theta_{n}(\alpha)^{c} \cap\left\{\|\theta\|_{1} \in\left(j \sqrt{n} \varepsilon_{n}(\alpha),(j+1) \sqrt{n} \varepsilon_{n}(\alpha)\right)\right\}, j \geq 0$. We use Lemma 5.b with $\alpha \geq 1 / 2+n^{-1 / 6}$, so that

$$
E\left(\|\theta\|_{1} \mid \alpha\right) \leq \sqrt{2 / \pi} \frac{2 \alpha}{2 \alpha-1}
$$


and from Lemma 3.2, $E\left(\|\theta\|_{1} \mid \alpha\right) \lesssim \sqrt{n} \varepsilon_{n}(\alpha)$. Also, for all $j \geq J_{1}$ with $J_{1}$ fixed and large enough following from Lemma 5.b we have

$$
\Pi\left(\|\theta\|_{1}>j \sqrt{n} \varepsilon_{n}(\alpha) \mid \alpha\right) \leq e^{-c_{0} j^{2} n \varepsilon_{n}^{2}(\alpha)},
$$

for some $c_{0}>0$ independent of $\alpha$. On $\Theta_{n, j}$ define $u_{n, j}=u_{n} /(j \log j)$ and construct a covering of $\left[\alpha, \alpha+u_{n}\right]$ with balls of radius $u_{n, j}$, the number of such balls is of order $N_{j}=O(j \log j)$. Then since

$$
\sup _{\left|\alpha-\alpha^{\prime}\right| \leq u_{n}} p_{\psi_{\alpha, \alpha^{\prime}}(\theta)}^{n}\left(\mathbf{x}_{n}\right) \leq \max _{i \leq N_{j}} \sup _{\left|\alpha^{\prime}-\alpha_{i}\right| \leq u_{n, j}} p_{\psi_{\alpha_{i}, \alpha^{\prime}}(\theta)}^{n}\left(\mathbf{x}_{n}\right)
$$

we have that for all $\theta \in B_{n, j}$ (where $B_{n, j}$ was defined in (A2 bis))

$$
Q_{\alpha, n}^{\theta}\left(\mathcal{X}^{n}\right) \leq N_{j} e^{2 a\|\varphi\|_{\infty} /(\log j)} \leq 2 N_{j},
$$

if $a$ is chosen small enough in the definition of $u_{n}$ and

$$
\int_{\Theta_{n, j}} Q_{\alpha, n}^{\theta}\left(\mathcal{X}^{n}\right) d \Pi(\theta \mid \alpha) \lesssim N_{j} \Pi\left(\Theta_{n, j} \mid \alpha\right) \lesssim j \log j e^{-c_{0} j^{2} n \varepsilon_{n}^{2}(\alpha)} .
$$

Let $j \leq J_{1}$, then $\|\theta\|_{1} \leq J_{1} \sqrt{n} \varepsilon_{n}(\alpha)$ and

$$
Q_{\alpha, n}^{\theta}\left(\mathcal{X}^{n}\right) \leq e^{c_{0} J_{1}^{2} n \varepsilon_{n}^{2}(\alpha) / 2}
$$

and, since by choosing $A$ (in the definition of $\left.\Theta_{n}(\alpha)\right)$ large enough $\Pi\left(\Theta_{n}^{c}(\alpha) \mid \alpha\right) \leq$ $e^{-c_{0} J_{1}^{2} n \varepsilon_{n}^{2}(\alpha)}$,

$$
\int_{\cup_{j \leq J_{1}} \Theta_{n, j}} Q_{\alpha, n}^{\theta}\left(\mathcal{X}^{n}\right) d \Pi(\theta \mid \alpha) \leq e^{c_{0} J_{1}^{2} n \varepsilon_{n}^{2}(\alpha) / 2} \Pi\left(\Theta_{n}^{c}(\alpha) \mid \alpha\right) \leq e^{-c_{0} J_{1}^{2} n \varepsilon_{n}^{2}(\alpha) / 2},
$$

which implies (2.6).

Similarly to the case of prior (T2), we verify (A2). The tests are the same as in Section 1.4, since $q_{\alpha, n}^{\theta} \lesssim f_{\theta}^{n}$ if $u_{n} \leq a n^{-3 / 2} \varepsilon_{n}(\alpha)^{-1}$ and the argument follows the same line, with $\varepsilon_{n}(\alpha)$ replacing $\varepsilon_{n}(\tau)$ and $\tilde{\Theta}_{n}(\alpha)$ replacing $\tilde{\Theta}_{n}(\tau)$ although the definitions remain the same. Note that in this case we do not have to split into $\tau$ large or small in the definition of $\bar{\Theta}$. Equation (1.j) is satisfied for all $\alpha \in(1 / 2, \log n /(16 \log \log n)]$ so that condition (A2) is verified.

The verification of $(\mathrm{C} 1)-(\mathrm{C} 3)$ follows the same lines as in the case of prior (T2) using the fact that if $\|\theta\|_{1} \leq M$, and if $u_{n} \leq n^{-3 / 2} \varepsilon_{n, 0} M_{n} /\left(M\|\varphi\|_{\infty}\right)$, for all $\theta \in \Theta_{n}(\alpha)$ and $\alpha \in \Lambda_{0}$,

$$
\inf _{\alpha \leq \alpha+u_{n}} \ell_{n}\left(\psi_{\alpha, \alpha^{\prime}}(\theta)\right)-\ell_{n}(\theta) \geq-1
$$


and

$$
\sup _{\alpha \leq \alpha+u_{n}} \ell_{n}\left(\psi_{\alpha, \alpha^{\prime}}(\theta)\right)-\ell_{n}(\theta) \leq 1
$$

The control over $\Theta_{n}(\alpha)^{c}$ is done as before by splitting it into the subsets $\Theta_{n, j}$. Finally similarly to the preceding sections $\log N_{n}\left(\Lambda_{n}\right)=o\left(n \varepsilon_{n, 0}^{2}\right)$ for arbitrarily small $c_{2}>0$.

1.6. Proof of Proposition 3.6. From [2], together with the fact that $q_{k, n}^{\theta}=$ $f_{\theta}^{\otimes n}$ for all $\theta \in \mathcal{S}_{k}$ (where $\mathcal{S}_{k}$ denotes the $k$ dimensional simplex) and that in $\mathcal{S}_{k}$ the set

$$
\left\{u \leq h\left(f_{0}, f_{\theta}\right) \leq 2 u\right\} \subset\left\{\sum_{j=1}^{k}\left(\sqrt{\theta_{j}}-\sqrt{\bar{\theta}_{j, k}}\right)^{2} \leq 8 u^{2}\right\}
$$

and the covering number of this set with balls of radius $\zeta u$ in Hellinger distance is bounded from above by $(C k / u \zeta)^{k}$ so that for all $u \geq A \sqrt{k / n}$, condition $(2.9)$ is verified. Finally since $f_{0}$ is bounded from above and from below the Kullback-Leiber divergence is bounded by a constant times the square of the Hellinger distance and condition (B1) is verified. Conditions (C1)-(C3) follow from the above arguments and the remark that when $f_{0} \in$ $\mathcal{H}_{\infty}(\beta, L)$ then $\Lambda_{0} \subset\left\{k \leq k_{1}(n / \log n)^{1 /(2 \beta+1)}\right\}$ for some $k_{1}$ large enough, as in the case of prior (T1).

1.7. Proof of Theorem 2.2. Similarly to the proof of Corollary 2.1 we can write

$$
\begin{aligned}
E_{\theta_{0}}^{n} \Pi\left(\theta: d\left(\theta, \theta_{0}\right) \leq \delta_{n} \varepsilon_{n, 0} \mid \mathbf{x}_{n}, \hat{\lambda}_{n}\right) & =E_{\theta_{0}}^{n}\left(\frac{G_{n}\left(\hat{\lambda}_{n}\right)}{m\left(\mathbf{x}_{n} \mid \hat{\lambda}_{n}\right)}\right) \\
& \leq e^{c_{2} n \varepsilon_{n, 0}^{2},} E_{\theta_{0}}^{n} \sup _{\lambda \in \Lambda_{0}} G_{n}(\lambda)+o(1),
\end{aligned}
$$

where $G_{n}(\lambda)=\int_{\theta: d\left(\theta, \theta_{0}\right) \leq \delta_{n} \varepsilon_{n, 0}} e^{\ell_{n}(\theta)-\ell_{n}\left(\theta_{0}\right)} d \Pi(\theta \mid \lambda)$.

Then similarly to the proof of Theorem 2.1 we take a $u_{n}$ covering of the set $\Lambda_{0}$ with center points $\lambda_{1}, \lambda_{2}, \ldots, \lambda_{N\left(\Lambda_{0}\right)}$ and we get (from conditions (A1) and (2.12)) that

$$
\begin{aligned}
E_{\theta_{0}}^{n} \sup _{\lambda \in \Lambda_{0}} G_{n}(\lambda) & =E_{\theta_{0}}^{n} \sup _{\lambda_{i}} \sup _{\rho\left(\lambda, \lambda_{i}\right) \leq u_{n}} \int_{d\left(\psi_{\lambda_{i}, \lambda}(\theta), \theta_{0}\right) \leq \delta_{n} \varepsilon_{n, 0}} e^{\ell_{n}\left(\psi_{\lambda_{i}, \lambda}(\theta)\right)-\ell_{n}\left(\theta_{0}\right)} d \Pi\left(\theta \mid \lambda_{i}\right) \\
& \leq \sum_{i=1}^{N\left(\Lambda_{0}\right)} \int_{\left\{d\left(\theta, \theta_{0}\right) \leq 2 \delta_{n} \varepsilon_{n, 0}\right\} \cup \Theta_{n}^{c}} Q_{\lambda, n}^{\theta}\left(\mathcal{X}^{n}\right) d \Pi\left(\theta \mid \lambda_{i}\right) \\
& \leq N\left(\Lambda_{0}\right)\left(e^{-\tilde{w}_{n}^{2} n \varepsilon_{n, 0}^{2}+e^{-w_{n}^{2} n \varepsilon_{n, 0}^{2}}}\right),
\end{aligned}
$$


for some $\tilde{w}_{n} \rightarrow \infty$, where in the first inequality we applied that following the (adjusted) condition (C3) and triangle inequality $\left\{\theta: d\left(\psi_{\lambda_{i}, \lambda}(\theta), \theta_{0}\right) \leq\right.$ $\left.\delta_{n} \varepsilon_{n, 0}\right\} \subset\left\{d\left(\theta, \theta_{0}\right) \leq 2 \delta_{n} \varepsilon_{n, 0}\right\} \cup \Theta_{n}^{c}$. We conclude the proof by combining the two displays.

\section{Some technical Lemmas for priors (T2) and (T3) .}

Lemma 2.a. For every $\alpha, \tau>0$, and $\zeta \in(0,1)$, take $\eta \geq \tilde{c}_{1}^{2}\left(3 \zeta^{-1} K / c\right)^{1 / \alpha}$ (with $c=c(\alpha, \tau)$ and $\tilde{c}_{1}$ given in (3.4)) and define the sets

$$
\Theta_{n}(\alpha, \tau)=(\zeta c / 3) \varepsilon_{n} \mathbb{B}_{1}+R_{n} \mathbb{H}_{1}^{\alpha, \tau}, \quad \varepsilon_{n}=\varepsilon_{n}(\alpha, \tau), \quad R_{n}=R_{n}(\alpha, \tau)
$$

with

$$
R_{n}=-2 \Phi^{-1}\left(e^{-\eta n \varepsilon_{n}^{2}}\right)
$$

and where $\mathbb{B}_{1} \subset \mathbb{R}^{n}$, respectively $\mathbb{H}_{1}^{\alpha, \tau}$, denotes the unit ball on the Hilbert space $\left(\mathbb{R}^{n},\|\cdot\|_{2}\right)$, respectively the reproducing kernel Hilbert space corresponding to the priors (T2) and (T3). Then

$$
\begin{array}{r}
\log N\left(c \zeta \varepsilon_{n}, \Theta_{n}(\alpha, \tau),\|\cdot\|_{2}\right) \leq 5 \eta n \varepsilon_{n}^{2}, \\
\Pi\left(\Theta_{n}^{c}(\alpha, \tau) \mid \alpha, \tau\right) \leq e^{-\eta n \varepsilon_{n}^{2}}, \text { and } \\
\left\|\Theta_{n}(\alpha, \tau)\right\|_{2}^{2} \leq 2^{4} \eta \tau^{2} n \varepsilon_{n}^{2} \vee 1 .
\end{array}
$$

Moreover, if $\alpha>1 / 2$, then for all $u_{n} / \tau<1$,

$$
\begin{gathered}
\log \Pi\left(\|\theta\|_{1} \leq u_{n} \mid \alpha, \tau\right) \geq-C_{\alpha}\left(\frac{u_{n}}{\tau}\right)^{-1 /(\alpha-1 / 2)} \\
C_{\alpha} \lesssim((\alpha-1 / 2) / 8 \varphi(0))^{-1 /(\alpha-1 / 2)} .
\end{gathered}
$$

Let $\mathbb{B}_{1}^{L_{1}}$ denote the ball of radius 1 centered at 0 for the norm $L_{1}$ and if

$$
\bar{\Theta}_{n}(\alpha, \tau)=u_{n} \mathbb{B}_{1}^{L_{1}}+\bar{R}_{n} \mathbb{H}_{1}^{\alpha, \tau}, \quad \bar{R}_{n}=-2 \Phi^{-1}\left(e^{-C_{\alpha}\left(\frac{u_{n}}{\tau}\right)^{-1 /(\alpha-1 / 2)}}\right)
$$

then

$$
\Pi\left(\bar{\Theta}_{n}^{c} \mid \alpha, \tau\right) \leq e^{-C C_{\alpha}\left(\frac{u_{n}}{\tau}\right)^{-1 /(\alpha-1 / 2)}}
$$

for some $C>0$ independent of $\alpha$ and $\tau$.

Proof. We follow the lines of the proof of Theorem 2.1 of [7]. Define $\gamma_{n}$ such that

$$
\Phi\left(\gamma_{n}\right) \equiv \Pi\left(\theta:\|\theta\|_{2} \leq \zeta c \varepsilon_{n} / 3 \mid \alpha, \tau\right) \geq e^{\eta \log \Pi\left(\theta:\left\|\theta-\theta_{0}\right\|_{2} \leq K \varepsilon_{n} \mid \alpha, \tau\right)}=e^{-\eta n \varepsilon_{n}^{2}},
$$


where $\Phi(x)$ denotes the distribution function of the standard normal random variable. Then we can see that $\gamma_{n} \geq-R_{n} / 2$ and therefore by Borell's inequality we have that

$$
\Pi\left(\Theta_{n}^{c} \mid \alpha, \tau\right) \leq 1-\Phi\left(\gamma_{n}+R_{n}\right) \leq 1-\Phi\left(R_{n} / 2\right)=e^{-\eta n \varepsilon_{n}^{2}} .
$$

Then take a $(2 \zeta c / 3) \varepsilon_{n}$-separated $h_{1}, h_{2}, \ldots, h_{N}$ points contained in $R_{n} \mathbb{H}_{1}$ for the $\|\cdot\|_{2}$ norm, so the $h_{i}+(\zeta c / 3) \varepsilon_{n}$-balls are separated. Furthermore note that following from the tail bound on the Gaussian distribution function $\Phi(-x) \leq e^{-x^{2} / 2} /(\sqrt{2 \pi} x) \leq e^{-x^{2} / 2}$ we have that

$$
R_{n}=-2 \Phi^{-1}\left(e^{-\eta n \varepsilon_{n}^{2}}\right) \leq \sqrt{8 \eta n \varepsilon_{n}^{2}}
$$

Then similarly to [7] (with $C=\eta$ and $\varphi_{0, \alpha, \tau}\left(\zeta c \varepsilon_{n} / 3\right) \leq \eta n \varepsilon_{n}^{2}$ ) we get that

$$
1 \geq N e^{-R_{n}^{2} / 2} e^{-\varphi_{0, \alpha, \tau}\left(c \varepsilon_{n} / 6\right)} \geq N e^{-5 \eta n \varepsilon_{n}^{2}} .
$$

This leads to the inequality

$$
\begin{aligned}
\log N\left(\zeta c \varepsilon_{n}, \Theta_{n}(\alpha, \tau),\|\cdot\|_{2}\right) & \leq \log N\left(2 \zeta c \varepsilon_{n} / 3, R_{n} \mathbb{H}_{1},\|\cdot\|_{2}\right) \\
& \leq \log N \leq 5 \eta n \varepsilon_{n}^{2}
\end{aligned}
$$

Finally we note that

$$
\left\|\Theta_{n}\right\|_{2}^{2} \leq\left(\tau R_{n}+(K / 6) \varepsilon_{n}\right)^{2} \leq 2 \tau^{2} R_{n}^{2} \vee 1 \leq 2^{4} \eta \tau^{2} n \varepsilon_{n}^{2} \vee 1
$$

Let $\alpha>1 / 2$, then the above argument implies that with $\bar{R}_{n}$ defined as in (2.c),

$$
\Pi\left(\Theta_{n}^{c} \mid \alpha, \tau\right) \leq 1-\Phi\left(\bar{R}_{n} / 2\right) \leq e^{-C_{\alpha}\left(u_{n} / \tau\right)^{-1 /(\alpha-1 / 2)}} .
$$

Also

$$
\begin{aligned}
& \Pi\left(\theta:\|\theta\|_{1} \leq u_{n} \mid \alpha, \tau\right) \\
& \quad=\Pi\left(\theta:\|\theta\|_{1} \leq u_{n} / \tau \mid \alpha, 1\right) \\
& \quad \geq\left(1-P\left(\sum_{j=J_{1}+1}^{\infty} j^{-\alpha-1 / 2}\left|Z_{j}\right|>u_{n} /(2 \tau)\right)\right) \prod_{j \leq J_{1}} P\left(j^{-\alpha-1 / 2}\left|Z_{j}\right| \leq \frac{u_{n}}{2 \tau J_{1}}\right)
\end{aligned}
$$

we choose $J_{1}$ such that the first factor is bounded from below by $1 / 2$. To do 
so we bound from above

$$
\begin{aligned}
P\left(\sum_{j=J_{1}+1}^{\infty} j^{-\alpha-1 / 2}\left|Z_{j}\right|\right. & \left.>u_{n} /(2 \tau)\right) \leq e^{-s u_{n} /(2 \tau)} \prod_{j=J_{1}+1}^{\infty} E\left(e^{s j^{-\alpha-1 / 2}\left|Z_{j}\right|}\right) \\
& =e^{-s u_{n} /(2 \tau)} e^{\frac{s^{2}}{2} \sum_{j \geq J_{1}+1} j^{-2 \alpha-1}} \prod_{j \geq J_{1}+1}\left(2 \Phi\left(s j^{-\alpha-1 / 2}\right)\right) \\
& \leq e^{-s u_{n} /(2 \tau)} e^{\frac{s^{2} J_{1}^{-2 \alpha}}{4 \alpha}} e^{2 s \varphi(0) \sum_{j \geq J_{1}+1} j^{-\alpha-1 / 2}} \\
& \leq \exp \left(-s\left(\frac{u_{n}}{2 \tau}-\frac{2 \varphi(0) J_{1}^{-\alpha+1 / 2}}{\alpha-1 / 2}\right)+\frac{s^{2} J_{1}^{-2 \alpha}}{4 \alpha}\right)
\end{aligned}
$$

We choose $J_{1}^{-\alpha+1 / 2} \leq(\alpha-1 / 2) \frac{u_{n}}{8 \varphi(0) \tau}$ so that for all $s>0$

$$
\begin{aligned}
P\left(\sum_{j=J_{1}+1}^{\infty} j^{-\alpha-1 / 2}\left|Z_{j}\right|>u_{n} /(2 \tau)\right) & \leq \exp \left(-s \frac{u_{n}}{4 \tau}+\frac{s^{2} J_{1}^{-2 \alpha}}{4 \alpha}\right) \\
& \leq \exp \left(-\frac{\alpha J_{1}^{2 \alpha} u_{n}^{2}}{8 \tau^{2}}\right),
\end{aligned}
$$

where the last inequality comes from choosing $s=\left(u_{n} / \tau\right) \alpha J_{1}^{2 \alpha}$. This probability is smaller than $1 / 2$ as soon as $\alpha J_{1}^{2 \alpha} u_{n}^{2} /\left(8 \tau^{2}\right) \geq \log 2$, i.e. as soon as $J_{1} \geq(8 \log 2 / \alpha)^{1 /(2 \alpha)}\left(u_{n} / \tau\right)^{-1 / \alpha}$. Since $\alpha>1 / 2$, there exists a constant $J_{0}$ such that both constraints are satisfied as soon as $J_{1} \geq J_{0}((\alpha-$ $1 / 2) / 8 \varphi(0))^{-1 /(\alpha-1 / 2)}\left(u_{n} / \tau\right)^{-1 /(\alpha-1 / 2)}$. We can then bound from below

$$
\prod_{j \leq J_{1}} P\left(j^{-\alpha-1 / 2}\left|Z_{j}\right| \leq \frac{u_{n}}{2 \tau J_{1}}\right)=\prod_{j \leq J_{1}}\left(2 \Phi\left(\frac{j^{\alpha+1 / 2} u_{n}}{2 \tau J_{1}}\right)-1\right) .
$$

For all $j^{\alpha+1 / 2} u_{n} \leq 2 \tau J_{1}$

$$
\left(2 \Phi\left(\frac{j^{\alpha+1 / 2} u_{n}}{2 \tau J_{1}}\right)-1\right) \geq 2 \frac{j^{\alpha+1 / 2} u_{n} \varphi(1)}{2 \tau J_{1}}
$$

for all $j^{\alpha+1 / 2} u_{n}>2 \tau J_{1}$

$$
\left(2 \Phi\left(\frac{j^{\alpha+1 / 2} u_{n}}{2 \tau J_{1}}\right)-1\right) \geq 2 \Phi(1)-1=C
$$

which leads to

$$
\prod_{j \leq J_{1}} P\left(j^{-\alpha-1 / 2}\left|Z_{j}\right| \leq \frac{u_{n}}{2 \tau J_{1}}\right) \geq \exp \left(-J_{1} c\right),
$$


for some $c$ independent of $\alpha$ and $\tau$.

Next we show that for the scaling prior (T2) in the case $\alpha+1 / 2 \leq \beta$ the second part of condition (2.12) holds.

Lemma 2.b. For the prior (T2), when $\alpha+1 / 2 \leq \beta$, then $\varepsilon_{n}(\tau) \geq n^{-\frac{2 \alpha+1}{4 \alpha+4}}$ for all $\tau \in \Lambda_{n}$ and for all $\theta_{0} \in \mathcal{H}_{\infty}(\beta, L) \cup \mathcal{S}_{\beta}(L), \theta_{0} \neq 0$ and $\delta_{n}=o\left(M_{n}^{-2}\right)$ we have

$$
\sup _{\tau \in \Lambda_{0}} \frac{n \varepsilon_{n}(\tau)^{2}}{-\log \Pi\left(\left\{\left\|\theta-\theta_{0}\right\|_{2} \leq \delta_{n} \varepsilon_{n}(\tau)\right\} \mid \tau, \alpha\right)}=o(1)
$$

Proof. First of all note that $\varepsilon_{n}(\tau) \geq n^{-(2 \alpha+1) /(4 \alpha+4)}$ follows automatically from (A.2). Then for every $\tau \in \Lambda_{0}$

$$
\begin{aligned}
-\log \Pi\left(\left\|\theta-\theta_{0}\right\|_{2} \leq \delta_{n} \varepsilon_{n}(\tau) \mid \tau, \alpha\right) & \geq-\log \Pi\left(\|\theta\|_{2} \leq \delta_{n} \varepsilon_{n}(\tau) \mid \tau, \alpha\right) \\
& \gtrsim \delta_{n}^{-1 / \alpha}\left(\varepsilon_{n}(\tau) / \tau\right)^{-1 / \alpha} \\
& \geq\left(M_{n}^{2} \delta_{n}\right)^{-1 / \alpha} \varepsilon_{n, 0}^{-1 / \alpha} n^{-\frac{1}{\alpha(4+4 \alpha)}}
\end{aligned}
$$

hence for $\delta_{n}=o\left(M_{n}^{-2}\right)$ following from Lemma 3.3 the right hand side of the preceding display is of higher order than $n \varepsilon_{n, 0}^{2}$.

3. Some technical Lemmas for the hyper-prior distributions. In this section we collect the proofs of the technical lemmas on the hyper-prior distribution.

3.1. Proof of Lemma 3.4. Take any $k_{0}$ satisfying $\varepsilon_{n}\left(k_{0}\right) \leq 2 \varepsilon_{n, 0}$. Since from the proof of Lemma $3.1 n \varepsilon_{n}(k)^{2}>k \log \sqrt{k}(1+o(1))$ holds we get

$$
\tilde{\pi}\left(k_{0}\right) \gtrsim k_{0}^{-c_{2} k_{0}} \geq e^{-2 c_{0} n \varepsilon_{n}^{2}\left(k_{0}\right)} \geq e^{-\tilde{w}_{n}^{2} n \varepsilon_{n, 0}^{2}} .
$$

For the upper bound we note that following from Lemma 3.1

$$
\begin{aligned}
\sum_{k=\varepsilon(n / \log n)}^{\infty} \tilde{\pi}(k) & \lesssim \sum_{k=\varepsilon(n / \log n)}^{\infty} e^{-c_{1} k^{\frac{1}{1+2 \beta_{0}}}} \lesssim e^{-c_{3}(n / \log n)^{\frac{1}{1+2 \beta_{0}}}} \\
& \lesssim e^{-\tilde{w}_{n}^{2} n^{\frac{1}{1+2 \beta}}} \leq e^{-\tilde{w}_{n}^{2} n \varepsilon_{n, 0}^{2}}
\end{aligned}
$$


3.2. Proof of Lemma 3.5. As a first step choose an arbitrary $\tau_{0} \in \Lambda_{0}\left(\tilde{w}_{n}\right)$ with $\varepsilon_{n}\left(\tau_{0}\right) \leq 2 \varepsilon_{n, 0}$. Then any $\tau$ satisfying $\varepsilon_{n}(\tau) \leq 2 \varepsilon_{n}\left(\tau_{0}\right)$ belongs to the set $\Lambda_{0}\left(\tilde{w}_{n}\right)$. Next consider $\tau$ satisfying $\varepsilon_{n}(\tau) \geq 2 \varepsilon_{n}\left(\tau_{0}\right)$. Furthermore, note that for any $\tau_{1}, \tau_{2}>0$ the RKHSs corresponding to the priors $\Pi\left(\cdot \mid \tau_{1}\right), \Pi\left(\cdot \mid \tau_{2}\right)$ are the same, i.e. $\mathbb{H}^{\tau_{1}}=\mathbb{H}^{\tau_{2}}$. Following from the definition of the concentration inequality $(3.2)$

$$
\begin{aligned}
& -\log \Pi\left(\left\|\theta-\theta_{0}\right\|_{2} \leq K \varepsilon_{n}(\tau) \mid \tau\right) \\
& \inf _{h \in \mathbb{H}^{\tau}:\left\|h-\theta_{0}\right\|_{2} \leq K \varepsilon_{n}(\tau) / 2}\|h\|_{\mathbb{H}^{\tau}}^{2}-\log \Pi\left(\|\theta\|_{2} \leq(K / 2) \varepsilon_{n}(\tau) \mid \tau\right) \\
& \leq \inf _{h \in \mathbb{H}^{\tau} \tau_{0}:\left\|h-\theta_{0}\right\|_{2} \leq K \varepsilon_{n}\left(\tau_{0}\right)}\left(\tau_{0} / \tau\right)^{2}\|h\|_{\mathbb{H}^{\tau_{0}}}^{2}-\log \Pi\left(\|\theta\|_{2} \leq\left(\tau_{0} / \tau\right) K \varepsilon_{n}\left(\tau_{0}\right) \mid \tau_{0}\right) \\
& \lesssim \max \left\{\left(\frac{\tau_{0}}{\tau}\right)^{2},\left(\frac{\tau_{0}}{\tau}\right)^{-\alpha}\right\}\left(\inf _{\substack{h \in \mathbb{H}^{\tau} \tau_{0} \\
\left\|h-\theta_{0}\right\|_{2} \leq K \varepsilon_{n}\left(\tau_{0}\right)}}\|h\|_{\mathbb{H}^{\tau_{0}}}^{2}-\log \Pi\left(\|\theta\|_{2} \leq K \varepsilon_{n}\left(\tau_{0}\right) \mid \tau_{0}\right)\right) \\
& \leq-\max \left\{\left(\tau_{0} / \tau\right)^{2},\left(\tau_{0} / \tau\right)^{-\alpha}\right\} \log \Pi\left(\left\|\theta-\theta_{0}\right\|_{2} \leq K \varepsilon_{n}\left(\tau_{0}\right) \mid \tau_{0}\right) \\
& \leq \max \left\{\left(\tau_{0} / \tau\right)^{2},\left(\tau_{0} / \tau\right)^{-\alpha}\right\} n \varepsilon_{n}\left(\tau_{0}\right)^{2}
\end{aligned}
$$

Hence $\varepsilon_{n}(\tau)^{2} \leq \max \left\{\left(\tau_{0} / \tau\right)^{2},\left(\tau_{0} / \tau\right)^{-\alpha}\right\} \varepsilon_{n}\left(\tau_{0}\right)^{2}$, so one can conclude that $\left[\left(2 / \tilde{w}_{n}\right) \tau_{0},\left(\tilde{w}_{n} / 2\right)^{2 / \alpha} \tau_{0}\right] \cap \Lambda_{n} \subset \Lambda_{0}\left(\tilde{w}_{n}\right)$. Therefore following from the proof of Lemma 3.2 we have $n \varepsilon_{n}(\tau)^{2} \geq \tau^{2 /(1+2 \alpha)} \vee \tau^{-2}$ and that $\left[\tau_{0} / 2,2 \tau_{0}\right] \in \Lambda_{n}$, hence

$$
\int_{\tau_{0} / 2}^{2 \tau_{0}} \tilde{\pi}(\tau) d \tau \gtrsim e^{-c_{1} \tau_{0}^{2 /(1+2 \alpha)}} \wedge e^{-c_{3} \tau_{0}^{-2}} \geq e^{-\tilde{w}_{n}^{2} n \varepsilon_{n, 0}^{2}} .
$$

concluding the first part of (H1).

The second assumption in (H1) holds trivially for $\tilde{\pi}$ satisfying the upper bounds in the lemma:

$$
\int_{0}^{e^{-c_{0} \bar{c}_{0} \tilde{w}_{n}^{2} n \varepsilon_{n, 0}^{2}}} \tilde{\pi}(\tau) d \tau \lesssim e^{-\bar{c}_{0} \tilde{w}_{n}^{2} n \varepsilon_{n, 0}^{2}}, \quad \int_{e^{c_{0} \bar{c}_{0} \tilde{w}_{n}^{2} n \varepsilon_{n, 0}^{2}}}^{\infty} \tilde{\pi}(\tau) d \tau \lesssim e^{-\bar{c}_{0} \tilde{w}_{n}^{2} n \varepsilon_{n, 0}^{2}}
$$

3.3. Proof of Remark 3.3. One can easily see that

$$
N_{n}\left(\Lambda_{n}\right) \leq 2 e^{c_{0} \bar{c}_{0} \tilde{w}_{n}^{2} n \varepsilon_{n, 0}^{2}} / e^{-2 c_{0} \bar{c}_{0} \tilde{w}_{n}^{2} n \varepsilon_{n, 0}^{2}} \lesssim e^{3 c_{0} \bar{c}_{0} \tilde{w}_{n}^{2} n \varepsilon_{n, 0}^{2}} .
$$

Then by noting that $\tilde{w}_{n}=o\left(w_{n}\right)$ we get our statement. The upper bound on the hyper-entropy of the set $\Lambda_{0}$ follows immediately from the proof of Proposition 3.2.

3.4. Proof of Lemma 3.6. Similarly to the proof of Lemma 3.6 we choose an arbitrary $\alpha_{0} \in \Lambda_{0}\left(\tilde{w}_{n}\right)$ with $\varepsilon_{n}\left(\alpha_{0}\right) \leq C \varepsilon_{n, 0}$. From Lemma 3.2 we have 
that $\alpha_{0} \geq \beta+o(1)$ in case $\theta_{0} \in \mathcal{S}^{\beta}(L) \cup \mathcal{H}(\beta, L)$, since $n^{-\beta /(1+2 \beta)} \gtrsim \varepsilon_{n}\left(\alpha_{0}\right) \gtrsim$ $n^{-\alpha_{0} /\left(1+2 \alpha_{0}\right)}$.

First assume that $\alpha_{0} \leq \log n$, then for any $\alpha \in\left[\alpha_{0} /\left(1+2 \log \tilde{w}_{n} / \log n\right), \alpha_{0}\right]$ we have that

$$
\begin{aligned}
-\log \Pi\left(\left\|\theta-\theta_{0}\right\|_{2} \leq K \varepsilon_{n}(\alpha) \mid \alpha\right) & \\
& \lesssim \inf _{h \in \mathbb{H}^{\alpha} 0:\left\|h-\theta_{0}\right\| \leq K \varepsilon_{n}\left(\alpha_{0}\right)}\|h\|_{\mathbb{H}^{\alpha} \alpha_{0}}^{2}+\left\{K \varepsilon_{n}\left(\alpha_{0}\right)\right\}^{-1 / \alpha} \\
& \lesssim\left(\inf _{h \in \mathbb{H}^{\alpha_{0}}:\left\|h-\theta_{0}\right\| \leq K \varepsilon_{n}\left(\alpha_{0}\right)}\|h\|_{\mathbb{H}^{\alpha_{0}}}^{2}-\log \Pi\left(\|\theta\|_{2} \leq K \varepsilon_{n}\left(\alpha_{0}\right) \mid \alpha_{0}\right)\right)^{\alpha_{0} / \alpha} \\
(3 . \mathrm{a}) & \leq\left\{n \varepsilon_{n}\left(\alpha_{0}\right)^{2}\right\}^{\alpha_{0} / \alpha} \leq \tilde{w}_{n}^{2} n \varepsilon_{n}\left(\alpha_{0}\right)^{2}
\end{aligned}
$$

hence $\left[\alpha_{0} /\left(1+2 \log \tilde{w}_{n} / \log n\right), \alpha_{0}\right] \subset \Lambda_{0}$. Then the first part of condition (H1) holds for $\tilde{\pi}$ satisfying the lower bound in the statement, since

$$
\int_{\alpha_{0} /\left(1+2 \log \tilde{w}_{n} / \log n\right)}^{\alpha_{0}} \tilde{\pi}(\alpha) d \alpha \gtrsim \frac{\alpha_{0} \log \tilde{w}_{n}}{(\log n) e^{c_{2} \alpha_{0}}} \gtrsim e^{-2 c_{2} \log n} \geq e^{-\tilde{w}_{n}^{2} n \varepsilon_{n, 0}^{2}},
$$

where in the last inequality we used that by definition $\varepsilon_{n, 0}^{2} \geq n^{-1} \log n$. In case $(\log n) / 2 \leq \alpha \leq \log n \leq \alpha_{0}$ we have that $\varepsilon_{n}\left(\alpha_{0}\right)^{-1 / \alpha} \lesssim n^{1 /(2 \alpha)} \lesssim 1$ hence similarly to (3.a) we have that $\alpha \in \Lambda_{0}$ so the statement follows from (3.b) with $\alpha_{0}$ replaced by $\log n$.

Finally we show that the second assumption in (H1) also holds if the upper bound on $\tilde{\pi}$ is satisfied

$$
\int_{c_{0} n^{c_{1}}}^{\infty} \tilde{\pi}(\alpha) d \alpha \lesssim e^{-c_{0} n} \lesssim e^{-\tilde{w}_{n}^{2} n \varepsilon_{n, 0}^{2}}
$$

if $\tilde{w}_{n} \leq c_{0}^{1 / 2} / \varepsilon_{n, 0}$.

\section{Some technical Lemmas for the nonparametric regression model.}

LEMMA 4.a. For $\theta_{0} \in \ell_{2}(M)$ (or equivalently $f_{0} \in L_{2}(M)$ ) we have for $u_{n} \leq n^{-3 / 2} / \log (n)$

$$
\sup _{\alpha, \tau} \sup _{\theta \in \mathbb{R}^{n},\left\|\theta-\theta_{0}\right\|_{2} \leq \varepsilon_{n}(\alpha, \tau)} Q_{\alpha, \tau, n}^{\theta}\left(\mathcal{X}_{n}\right)=O(1) .
$$

Proof. Let us denote by $\lambda$ the hyper-parameters $\alpha$ or $\tau$, and by $\rho\left(\lambda, \lambda^{\prime}\right)$ the losses $\left|\alpha-\alpha^{\prime}\right|$ or $\left|\log \tau^{\prime}-\log \tau\right|$. Furthermore, we introduce the notations

$$
\underline{\psi}_{\lambda, i}^{\theta}=\inf _{\rho\left(\lambda, \lambda^{\prime}\right) \leq u_{n}} \sum_{j=1}^{n} \psi_{\lambda, \lambda^{\prime}}\left(\theta_{j}\right) e_{j}\left(t_{i}\right) ; \quad \bar{\psi}_{\lambda, i}^{\theta}=\sup _{\rho\left(\lambda, \lambda^{\prime}\right) \leq u_{n}} \sum_{j=1}^{n} \psi_{\lambda, \lambda^{\prime}}\left(\theta_{j}\right) e_{j}\left(t_{i}\right) .
$$


For instance in case of $\lambda=\alpha$ this is

$$
\underline{\psi}_{\alpha, i}^{\theta}=\sum_{j=1}^{n} j^{-\operatorname{sign}\left(\theta_{j} e_{j}\left(t_{i}\right)\right) u_{n}} \theta_{j} e_{j}\left(t_{i}\right) ; \quad \bar{\psi}_{\alpha, i}^{\theta}=\sum_{j=1}^{n} j^{\operatorname{sign}\left(\theta_{j} e_{j}\left(t_{i}\right)\right) u_{n}} \theta_{j} e_{j}\left(t_{i}\right),
$$

while for $\lambda=\tau$

$\underline{\psi}_{\tau, i}^{\theta}=e^{-\operatorname{sign}\left(\sum_{j=1}^{n} \theta_{j} e_{j}\left(t_{i}\right)\right) u_{n}} \sum_{j=1}^{n} \theta_{j} e_{j}\left(t_{i}\right) ; \bar{\psi}_{\tau, i}^{\theta}=e^{\operatorname{sign}\left(\sum_{j=1}^{n} \theta_{j} e_{j}\left(t_{i}\right)\right) u_{n}} \sum_{j=1}^{n} \theta_{j} e_{j}\left(t_{i}\right)$.

Then one can easily obtain (using Cauchy-Schwarz inequality) that both in the case of $\lambda=\alpha$ and $\lambda=\tau$ we have

$$
\begin{aligned}
\left|\underline{\psi}_{\lambda, i}^{\theta}-\bar{\psi}_{\lambda, i}^{\theta}\right| & \leq\left(n^{u_{n}}-n^{-u_{n}}\right) \sum_{j=1}^{n} \theta_{j} e_{j}\left(t_{i}\right) \leq 2 u_{n} n^{u_{n}} \log n \sum_{j=1}^{n}\left|\theta_{j} e_{j}\left(t_{i}\right)\right| \\
& \leq 2 M\|\theta\|_{2} u_{n} n^{u_{n}+1 / 2} \log n .
\end{aligned}
$$

Writing out the definition of $Q_{\lambda, n}^{\theta}$ :

$$
Q_{\lambda, n}^{\theta}=\int_{\mathbb{R}^{n}} \sup _{\rho\left(\lambda, \lambda^{\prime}\right) \leq u_{n}} \prod_{i=1}^{n} \frac{1}{\sqrt{2 \pi \sigma^{2}}} e^{-\frac{\left(x_{i}-\sum_{j=1}^{n} \psi_{\lambda, \lambda^{\prime}}\left(\theta_{j}\right) e_{j}\left(t_{i}\right)\right)^{2}}{2 \sigma^{2}}} d \mathbf{x}_{n} .
$$

We deal with the one dimensional integrals separately

$$
\begin{aligned}
& \int_{\mathbb{R} \rho\left(\lambda, \lambda^{\prime}\right) \leq u_{n}} \frac{1}{\sqrt{2 \pi \sigma^{2}}} e^{-\frac{\left\{x_{i}-\sum_{j=1}^{n} \psi_{\lambda, \lambda^{\prime}}\left(\theta_{j}\right) e_{j}\left(t_{i}\right)\right\}^{2}}{2 \sigma^{2}}} d x_{i} \\
& \quad \leq \int_{x_{i}<\underline{\psi}_{\lambda, i}^{\theta} \cup x_{i}>\bar{\psi}_{\lambda, i}^{\theta}} \frac{1}{\sqrt{2 \pi \sigma^{2}}} e^{-\frac{\left(x_{i}-\underline{\psi}_{\lambda, i}^{\theta}\right)^{2}}{2 \sigma^{2}}} d x_{i}+\int_{\underline{\psi}_{\lambda, i}^{\theta}}^{\bar{\psi}_{\lambda, i}^{\theta}} \frac{1}{\sqrt{2 \pi \sigma^{2}}} d x_{i} \\
& \quad \leq \int_{\mathbb{R}} \frac{1}{\sqrt{2 \pi \sigma^{2}}} e^{-z^{2} /\left(2 \sigma^{2}\right)} d z+\frac{1}{\sqrt{2 \pi \sigma^{2}}}\left|\bar{\psi}_{\lambda, i}^{\theta}-\underline{\psi}_{\lambda, i}^{\theta}\right| \\
& \quad \leq 1+2 M\|\theta\|_{2} u_{n} \sigma^{-1} n^{u_{n}+1 / 2} \log n,
\end{aligned}
$$

where the last inequality follows from (4.b). Note that for $\theta_{0} \in \ell_{2}(M)$ and $\theta \in \mathbb{R}^{n}$ satisfying $\left\|\theta-\theta_{0}\right\|_{2} \leq \varepsilon_{n}$ we have

$$
\|\theta\|_{2} \leq\left\|\theta_{0}\right\|_{2}+\varepsilon_{n} \leq 2 M
$$

Therefore the right hand side of (4.c) is bounded from above by

$$
\left(1+2 M\|\theta\|_{2} u_{n} \sigma^{-1} n^{u_{n}+1 / 2} \log n\right)^{n} \leq e^{2 M\|\theta\|_{2} u_{n} \sigma^{-1} n^{u_{n}+3 / 2} \log n}=O(1) .
$$


Lemma 4.b. Consider priors (T2) and (T3). For $u_{n} \lesssim n^{-3} / \log n$ if $\lambda=\alpha$, and $u_{n} \lesssim \tau_{n}^{-2} \wedge n^{-5 / 2}$ if $\lambda=\tau<\tau_{n}$ we have that

$$
\int_{\Theta_{n}^{c}} Q_{\tau, \alpha, n}^{\theta}\left(\mathcal{X}_{n}\right) \Pi(d \theta \mid \tau, \alpha) \leq 3 e^{-(\eta / 2) n \varepsilon_{n}^{2}}
$$

where $\varepsilon_{n}=\varepsilon(\tau)$ or $\varepsilon_{n}(\alpha)$ and $\eta \geq \tilde{c}_{2}(12 K / c)^{1 / \alpha}$.

Proof. Following the proof of Lemma 4.a and using Cauchy-Schwarz inequality we get that

$$
\begin{aligned}
& \int_{\Theta_{n}^{c}} Q_{\tau, \alpha, n}^{\theta}\left(\mathcal{X}_{n}\right) \Pi(d \theta \mid \tau, \alpha) \leq \int_{\Theta_{n}^{c}} e^{2 M n^{1+u_{n}}(\log n) u_{n} \sum_{i=1}^{n}\left|\theta_{i}\right|} d \Pi(\theta \mid \tau, \alpha) \\
& \leq \Pi\left(\Theta_{n}^{c} \mid \tau, \alpha\right)^{1 / 2} \sqrt{\int_{\Theta} e^{2 M \sum_{i=1}^{n}\left|\theta_{i}\right| n^{1+u_{n}}(\log n) u_{n}} d \Pi(\theta \mid \tau, \alpha)} \\
& \leq e^{-(\eta / 2) n \varepsilon_{n}^{2}} 2 e^{M^{2} n^{2+2 u_{n}} \tau^{2}(\log n)^{2} u_{n}^{2} \sum_{i=1}^{n} i^{-1-2 \alpha}} \leq 3 e^{-(\eta / 2) n \varepsilon_{n}^{2}}
\end{aligned}
$$

LEMMA 4.c. In the nonparametric regression model for $\tau \leq \tau_{n}$ (for some $\left.\tau_{n} \rightarrow \infty\right), u_{n} \lesssim \tau_{n}^{-2} n^{-5 / 2} / \log n$, and $\theta \in \Theta_{n}(\alpha, \tau)$ (defined in (2.a)), there exist tests $\varphi_{n}(\theta)$ such that

$$
\sup _{\substack{\alpha>0 \\ \tau \leq \tau_{n}} \sup _{\substack{\theta^{\prime} \in \Theta_{n}(\alpha, \tau) \\\left\|\theta-\theta^{\prime}\right\|_{2}<\left\|\theta-\theta_{0}\right\|_{2} / 18}} \int_{\mathcal{X}_{n}}\left(1-\varphi_{n}(\theta)\right) d Q_{\alpha, \tau, n}^{\theta^{\prime}}\left(x^{(n)}\right) \leq e^{-\frac{1}{2} n\left\|\theta-\theta_{0}\right\|_{2}^{2}} .}
$$

Proof. First of all note that the likelihood ratio test (using the sequence notation)

$$
\varphi_{n}(\theta)=11\left[\sum_{i=1}^{n}\left\{x_{i}-\sum_{j=1}^{n} \theta_{j} e_{j}\left(t_{i}\right)\right\}^{2}-\sum_{i=1}^{n}\left\{x_{i}-\sum_{j=1}^{n} \theta_{0, j} e_{j}\left(t_{i}\right)\right\}^{2}\right] .
$$

satisfies for all $\theta \in \mathbb{R}^{n}$

$$
\sup _{\theta^{\prime} \in \mathbb{R}^{n}:\left\|\theta-\theta^{\prime}\right\|_{2} \leq\left\|\theta-\theta_{0}\right\|_{2} / 18} E_{\theta^{\prime}}^{n}\left(1-\varphi_{n}(\theta)\right) \leq \exp \left\{-\frac{n}{2}\left\|\theta-\theta_{0}\right\|_{2}^{2}\right\}
$$

and $E_{\theta_{0}}^{n} \varphi_{n}(\theta) \leq \exp \left\{-\frac{n}{2}\left\|\theta-\theta_{0}\right\|_{2}^{2}\right\}$, see for instance Section 7.7 of [4]. 
For notational convenience let us again denote by $\lambda$ both of the hyperparameters $\alpha$ and $\tau$, then we have that

$$
\begin{aligned}
& \int_{\mathbb{R}^{n}}\left(1-\varphi_{n}(\theta)\right) \sup _{\rho\left(\lambda, \lambda^{\prime}\right) \leq u_{n}} \frac{1}{\left(2 \pi \sigma^{2}\right)^{n / 2}} e^{-\frac{\left.\sum_{i=1}^{n}\left\{\sum_{j=1}^{n} \psi_{\lambda, \lambda^{\prime}}\left(\theta_{j}^{\prime}\right) e_{j}\left(t_{i}\right)-x_{i}\right)\right\}^{2}}{2 \sigma^{2}}} d \mathbf{x}_{n} \\
& \leq \int_{\left\|\mathbf{x}_{n}\right\|_{2} \leq \tau_{n} n}\left(1-\varphi_{n}(\theta)\right) \frac{1}{\left(2 \pi \sigma^{2}\right)^{n / 2}} e^{-\frac{\left.\sum_{i=1}^{n}\left\{\sum_{j=1}^{n} \theta_{j}^{\prime} e_{j}\left(t_{i}\right)-x_{i}\right)\right\}^{2}}{2 \sigma^{2}}} \\
& \quad \times \sup _{\rho\left(\lambda, \lambda^{\prime}\right) \leq u_{n}}\left(e^{\frac{1}{2 \sigma^{2}} \sum_{i=1}^{n}\left[\left\{\sum_{j=1}^{n} \theta_{j}^{\prime} e_{j}\left(t_{i}\right)-x_{i}\right\}^{2}-\left\{\sum_{j=1}^{n} \psi_{\lambda, \lambda^{\prime}}\left(\theta_{j}^{\prime}\right) e_{j}\left(t_{i}\right)-x_{i}\right\}^{2}\right]}\right) d \mathbf{x}_{n} \\
& \quad+\int_{\left\|\mathbf{x}_{n}\right\|_{2}>\tau_{n} n} \sup _{\rho\left(\lambda, \lambda^{\prime}\right) \leq u_{n}} \frac{1}{\left(2 \pi \sigma^{2}\right)^{n / 2}} e^{-\frac{1}{2 \sigma^{2}} \sum_{i=1}^{n}\left\{\sum_{j=1}^{n} \psi_{\lambda, \lambda^{\prime}}\left(\theta_{j}^{\prime}\right) e_{j}\left(t_{i}\right)-x_{i}\right\}^{2}} d \mathbf{x}_{n} .
\end{aligned}
$$

We deal with the two terms on the right hand side separately.

First we examine the first term, where it is enough to show that the multiplicative term (with the sup) is bounded from above by a constant. Using Cauchy-Schwarz and triangle inequalities and the assumption $\left|e_{j}\left(t_{i}\right)\right| \leq M$ we get that

$$
\begin{aligned}
& \sup _{\rho\left(\lambda, \lambda^{\prime}\right) \leq u_{n}}\left|\sum_{i=1}^{n}\left[\left\{\sum_{j=1}^{n} \theta_{j}^{\prime} e_{j}\left(t_{i}\right)-x_{i}\right\}^{2}-\left\{\sum_{j=1}^{n} \psi_{\lambda, \lambda^{\prime}}\left(\theta_{j}^{\prime}\right) e_{j}\left(t_{i}\right)-x_{i}\right\}^{2}\right]\right| \\
& \leq M \sqrt{\sum_{i=1}^{n}\left(\bar{\psi}_{\lambda, i}^{\theta^{\prime}}-\underline{\psi}_{\lambda, i}^{\theta^{\prime}}\right)^{2}}\left(2\left\|\mathbf{x}_{n}\right\|_{2}+M \sqrt{n}\left\|\theta^{\prime}\right\|_{2}+M \sqrt{n} \sup _{\rho\left(\lambda, \lambda^{\prime}\right) \leq u_{n}}\left\|\psi_{\lambda, \lambda^{\prime}}\left(\theta^{\prime}\right)\right\|_{2}\right)
\end{aligned}
$$

The right hand side of the preceding display following from (4.b) and $\left\|\mathbf{x}_{n}\right\|_{2} \leq$ $n$ is bounded from above by

$$
2 M^{2}\left\|\theta^{\prime}\right\|_{2} u_{n} n^{u_{n}+1}(\log n)\left(2 n \tau_{n}+C \sqrt{n}\left\|\theta^{\prime}\right\|_{2}\right) \lesssim u_{n} \tau_{n}^{2} n^{5 / 2} \log n=O(1) .
$$

Therefore it remained to deal with the second term on the right hand side of (4.g). Since $\left\|\theta^{\prime}\right\|_{2} \lesssim \sqrt{n} \tau_{n} \varepsilon_{n}$ (following from $\theta^{\prime} \in \Theta_{n}(\lambda)$ ) we have that $\sup _{\rho\left(\lambda, \lambda^{\prime}\right) \leq u_{n}}\left\|\psi_{\lambda, \lambda^{\prime}}\left(\theta^{\prime}\right)\right\| \leq n^{u_{n}}\left\|\theta^{\prime}\right\|_{2} \lesssim n^{u_{n}+1 / 2} \tau_{n} \varepsilon_{n}=o\left(n \tau_{n}\right)$. Therefore

$$
\begin{aligned}
\int_{\left\|\mathbf{x}_{n}\right\|_{2} \geq \tau_{n} n} & \frac{1}{\left(2 \pi \sigma^{2}\right)^{n / 2}} e^{-\frac{\sum_{i=1}^{n}\left(\sum_{j=1}^{n} \psi_{\lambda, \lambda^{\prime}}\left(\theta_{j}^{\prime}\right) e_{j}\left(t_{i}\right)-x_{i}\right)^{2}}{2 \sigma^{2}}} d \mathbf{x}_{n} \\
& \leq \int_{\left\|\mathbf{x}_{n}\right\|_{2} \geq \tau_{n} n} \frac{1}{\left(2 \pi \sigma^{2}\right)^{n / 2}} e^{-\frac{\left\|\mathbf{x}_{n}\right\|^{2} / 2}{2 \sigma^{2}}} d \mathbf{x}_{n} \leq 2^{n} e^{-n^{2} \tau_{n}^{2} /\left(2 \sigma^{2}\right)}
\end{aligned}
$$


where the right hand side is of smaller order than $\exp \left\{-(1 / 2) n\left\|\theta-\theta_{0}\right\|_{2}^{2}\right\}$, since $\left\|\theta-\theta_{0}\right\|_{2} \leq\left\|\theta_{0}\right\|_{2}+\|\theta\|_{2} \lesssim 1+\tau_{n} \sqrt{n} \varepsilon_{n}(\lambda)=o\left(\tau_{n} \sqrt{n}\right)$.

Lemma 4.d. Consider the nonparametric regression model and priors of type (T1)-(T3). Take any $\tau_{n} \rightarrow \infty$ and $0<\tau \leq \tau_{n}$. Then for $u_{n} \lesssim$ $n^{-2} \tau_{n}^{-1} / \log n$

$\sup _{\left\|\theta-\theta_{0}\right\| \leq K \varepsilon_{n}(\lambda)} P_{\theta_{0}}^{n}\left\{\inf _{\rho\left(\lambda, \lambda^{\prime}\right) \leq u_{n}} \ell_{n}\left(\psi_{\lambda, \lambda^{\prime}}(\theta)\right)-\ell_{n}\left(\theta_{0}\right) \leq-c_{3} n \varepsilon_{n}(\lambda)^{2}\right\}=e^{-n \varepsilon_{n}(\lambda)^{2}}$

for $c_{3} \geq 2+3 \sigma^{-2} K^{2} / 2$.

Proof. By triangle inequality we have that

$$
\left.\left|\ell_{n}\left(\psi_{\lambda, \lambda^{\prime}}(\theta)\right)-\ell_{n}\left(\theta_{0}\right)\right| \leq\left|\ell_{n}\left(\psi_{\lambda, \lambda^{\prime}}(\theta)\right)-\ell_{n}(\theta)\right|+\mid \ell_{n}(\theta)\right)-\ell_{n}\left(\theta_{0}\right) \mid .
$$

We deal with the two terms on the right hand side separately.

First consider the first term on the right hand side of (4.j) and note that in case of prior (T1) it is zero. For priors (T2)-(T3) following from Lemma 4.c we have that for $\left\|\mathbf{x}_{n}\right\|_{2} \leq n \tau_{n}$ it is bounded above by a constant, while $P_{\theta_{0}}^{n}\left(\left\|\mathbf{x}_{n}\right\|_{2} \geq n \tau_{n}\right) \leq e^{-c n^{2} \tau_{n}^{2}}$.

For the second term on the right hand side of (4.j) we apply Chernoff's inequality and $K\left(\theta, \theta_{0}\right)=\sigma^{-2}\left\|\theta-\theta_{0}\right\|_{2}^{2}$

$$
\begin{aligned}
& \sup _{\theta \in\left\|\theta-\theta_{0}\right\|_{2} \leq K \varepsilon_{n}} P_{\theta_{0}}^{n}\left\{\ell_{n}(\theta)-\ell_{n}\left(\theta_{0}\right) \leq-\left(3 \sigma^{-2} K^{2} / 2+1\right) n \varepsilon_{n}^{2}\right\} \\
& \leq \sup _{\theta \in\left\|\theta-\theta_{0}\right\|_{2} \leq K \varepsilon_{n}} P_{\theta_{0}}^{n}\left[\ell_{n}(\theta)-\ell_{n}\left(\theta_{0}\right)-E_{\theta_{0}}^{n}\left\{\ell_{n}(\theta)-\ell_{n}\left(\theta_{0}\right)\right\} \leq-\frac{K^{2}+2 \sigma^{2}}{2 \sigma^{2}} n \varepsilon_{n}^{2}\right] \\
& \leq \sup _{\theta \in\left\|\theta-\theta_{0}\right\|_{2} \leq K \varepsilon_{n}} e^{-\left(\sigma^{-2} K^{2} / 2+1\right) n \varepsilon_{n}^{2}} E\left(e^{\sigma^{-2}\left\langle\sum_{j=1}^{n}\left(\theta_{0, j}-\theta_{j}\right) e_{j}, Z\right\rangle_{n}}\right) \\
& \leq \sup _{\theta \in\left\|\theta-\theta_{0}\right\|_{2} \leq K \varepsilon_{n}} e^{-\left(\sigma^{-2} K^{2} / 2+1\right) n \varepsilon_{n}^{2}} e^{\sigma^{-2}(n / 2)\left\|\theta_{0}-\theta\right\|_{2}^{2}} \leq e^{-n \varepsilon_{n}^{2}},
\end{aligned}
$$

where $Z$ denotes an $n$ dimensional vector of iid standard normal random variables.

\section{Some Technical Lemmas in the density case.}

Lemma 5.a. Let $f_{0}=f_{\theta_{0}}, f_{\theta}$ with $\left\|\theta-\theta_{0}\right\|_{1}<+\infty$, and $\theta, \theta_{0} \in \ell_{2}$, then

$$
\begin{aligned}
& K\left(f_{0}, f_{\theta}\right) \leq\left\|f_{0}\right\|_{\infty} e^{\left\|\theta-\theta_{0}\right\|_{1}\|\varphi\|_{\infty}}\left\|\theta-\theta_{0}\right\|_{2}^{2}, \\
& V_{2}\left(f_{0}, f_{\theta}\right) \leq\left\|f_{0}\right\|_{\infty}\left\|\theta-\theta_{0}\right\|_{2}^{2}, \\
& d^{2}\left(f_{0}, f_{\theta}\right) \gtrsim \exp \left(-c_{1}\left\|\theta-\theta_{0}\right\|_{1}\right)\left\|\theta-\theta_{0}\right\|_{2}^{2} .
\end{aligned}
$$


Proof. We have following [5], see also the supplement, Section 3.2, Proof of Proposition 5 of [2] if $\left\|\log f_{\theta}\right\|_{\infty} \leq M$

$$
K\left(f_{0}, f_{\theta}\right)=\left\langle\theta_{0}-\theta, \varphi\left(f_{0}\right)\right\rangle_{2}-c\left(\theta_{0}\right)+c(\theta),
$$

with

$$
\begin{aligned}
c(\theta)-c\left(\theta_{0}\right) & =\log \left(\int f_{0}(x) e^{\sum_{j}\left(\theta_{j}-\theta_{0, j}\right) \varphi_{j}(x)} d x\right) \\
& \leq 1-\left\langle\theta_{0}-\theta, \varphi\left(f_{0}\right)\right\rangle+\frac{\left\|f_{0}\right\|_{\infty}}{2} e^{\|\varphi\|_{\infty}\left\|\theta-\theta_{0}\right\|_{1}}\left\|\theta-\theta_{0}\right\|_{2}^{2} .
\end{aligned}
$$

Since $\left\langle\theta_{0}-\theta, \varphi\left(f_{0}\right)\right\rangle_{2}^{2} \leq\left\|f_{0}\right\|_{\infty}^{2}\left\|\theta-\theta_{0}\right\|_{2}^{2}$, this leads to

$$
K\left(f_{0}, f_{\theta}\right) \leq\left\|f_{0}\right\|_{\infty} e^{\|\varphi\|_{\infty}\left\|\theta-\theta_{0}\right\|_{1}}\left\|\theta-\theta_{0}\right\|_{2}^{2} .
$$

Similarly

$$
\begin{aligned}
V_{2}\left(f_{0}, f_{\theta}\right) & \leq 2\left(E_{\theta_{0}}\left[\left(\sum_{j}\left(\theta_{j}-\theta_{0, j}\right) \varphi_{j}\right)^{2}\right]+\left\langle\theta-\theta_{0}, \varphi\left(f_{0}\right)\right\rangle^{2}\right) \\
& \leq 2 c_{2}\left\|f_{0}\right\|_{\infty}^{2}\left\|\theta-\theta_{0}\right\|_{2}^{2} .
\end{aligned}
$$

Finally using the inequality $\left|e^{v}-e^{w}\right|=e^{v}\left|1-e^{w-v}\right| \geq e^{v} e^{-|w-v|}|w-v|$ and that $\log f_{0}>-\infty$ we have that $d\left(f_{0}, f_{\theta}\right)$ is bounded from below by

$$
\begin{aligned}
\int_{0}^{1} f_{0} e^{-\left|\sum_{j}\left(\theta_{0, j}-\theta_{j}\right) \varphi_{j}(x)+c(\theta)-c\left(\theta_{0}\right)\right|} & \left(\sum_{j}\left(\theta_{j}-\theta_{0, j}\right) \varphi_{j}(x)+c(\theta)-c\left(\theta_{0}\right)\right)^{2} d x \\
& \gtrsim e^{-2\|\varphi\|_{\infty}\left\|\theta-\theta_{0}\right\|_{1}}\left\|\theta-\theta_{0}\right\|_{2}^{2},
\end{aligned}
$$

where in the last inequality we applied the orthonormality of the basis $\varphi_{j}$ and the inequality $e^{\left|c(\theta)-c\left(\theta_{0}\right)\right|} \leq e^{\|\varphi\|_{\infty}\left\|\theta-\theta_{0}\right\|_{1}}$.

Lemma 5.b. Let $\Pi(\cdot \mid \alpha, \tau)$ be the Gaussian prior with $\alpha>1 / 2$ and $\tau \in$ $\left(n^{-a}, n^{b}\right)$ then

$$
\begin{array}{r}
E\left(\|\theta\|_{1} \mid \alpha, \tau\right)=\tau E\left(\|\theta\|_{1} \mid \alpha, \tau=1\right) \equiv \tau A_{\alpha}<\infty, \\
\Pi\left(\|\theta\|_{1}>t+\tau A_{\alpha} \mid \alpha, \tau\right) \leq e^{-\frac{t^{2}}{2 \sigma_{\alpha, \tau}^{2}}}
\end{array}
$$

with

$$
\sigma_{\alpha, \tau} \leq \sigma_{1 / 2,1} \tau, \quad \forall \alpha \geq 1 / 2, \tau>0 .
$$

Moreover, for all $K_{n}>0$ going to infinity and all $M>0$

$$
\Pi\left(\left\|\theta-\theta_{0}\right\|_{1}>M+\left\|\theta_{0}\right\|_{1}+\sqrt{K_{n}}\left\|\theta-\theta_{0}\right\|_{2} \mid \alpha, \tau\right) \leq e^{-\frac{M^{2} K_{n}^{2 \alpha}}{2 \tau^{2}}} .
$$


Proof. The first part of Lemma 5.b is Borell's inequality associated to the Banach space $\ell_{1}=\left\{\theta ; \sum_{i}\left|\theta_{i}\right|<+\infty\right\}$ since

$$
\Pi\left(\|\theta\|_{1}<+\infty \mid \alpha, \tau\right)=1, \quad \forall \alpha>1 / 2, \tau>0 .
$$

To prove (5.d), let $K_{n}>0$ then

$$
\left\|\theta-\theta_{0}\right\|_{1} \leq \sqrt{K_{n}}\left\|\theta-\theta_{0}\right\|_{2}+\sum_{j>K_{n}}\left|\theta_{0 j}\right|+\sum_{j>K_{n}}\left|\theta_{j}\right|
$$

and

$$
\Pi\left(\sum_{j>K_{n}}\left|\theta_{j}\right|>M \mid \alpha, \tau\right)=P\left(\sum_{j>K_{n}} j^{-\alpha-1 / 2}\left|Z_{j}\right|>M / \tau\right) \leq e^{-\frac{M^{2} K_{n}^{2 \alpha}}{2 \tau^{2}}},
$$

where $Z_{j} \stackrel{i i d}{\sim} \mathcal{N}(0,1)$.

LEMMA 5.c. In the density estimation problem with a prior on $f_{\theta}$ defined by (3.15), if there exists $M>0$ and $\varepsilon_{n}$ such that $n \varepsilon_{n}^{2} \rightarrow+\infty$, and

$$
\left\{\left\|\theta-\theta_{0}\right\|_{2} \leq \varepsilon_{n}\right\} \cap\left\{\|\theta\|_{1} \leq M\right\} \subset B_{n}\left(\theta_{0}, M_{2} \varepsilon_{n}, 2\right),
$$

then there exists $a_{0}>0$ such that for all $\left\{\left\|\theta-\theta_{0}\right\|_{2} \leq \varepsilon_{n}\right\} \cap\left\{\|\theta\|_{1} \leq M\right\}$,

$$
P_{\theta_{0}}^{n}\left(\ell_{n}(\theta)-\ell_{n}\left(\theta_{0}\right) \leq-2 M_{2} n \varepsilon_{n}^{2}\right) \leq e^{-a_{0} n \varepsilon_{n}^{2}}
$$

Proof. Let $\theta \in\left\{\left\|\theta-\theta_{0}\right\|_{2} \leq \varepsilon_{n}\right\} \cap\left\{\|\theta\|_{1} \leq M\right\}$,

$$
\begin{aligned}
P_{\theta_{0}}^{n}\left\{\ell_{n}(\theta)\right. & \left.-\ell_{n}\left(\theta_{0}\right) \leq-2 M_{2} n \varepsilon_{n}^{2}\right\} \\
& \leq e^{-2 s M_{2} n \varepsilon_{n}^{2}}\left(1+K\left(f_{0}, f_{\theta}\right)+V_{2}\left(f_{0}, f_{\theta}\right) e^{2 s\left\|\theta-\theta_{0}\right\|_{1}\|\varphi\|_{\infty}}\right)^{n} \\
& \leq e^{-a_{0} n \varepsilon_{n}^{2}}
\end{aligned}
$$

for some $a_{0}>0$ proportional to $M_{2}$.

\section{References.}

[1] Arbel, J., Gayraud, G., and Rousseau, J. (2013). Bayesian optimal adaptive estimation using a sieve prior. Scandinavian Journal of Statistics, 40(3):549-570.

[2] Castillo, I. and Rousseau, J. (2013). A general bernstein-von mises theorem in semiparametric models : Supplementary material. Ann. Statist.

[3] Ghosal, S., Ghosh, J. K., and van der Vaart, A. (2000). Convergence rates of posterior distributions. Ann. Statist., 28:500-531.

[4] Ghosal, S. and van der Vaart, A. (2007). Convergence rates of posterior distributions for non iid observations. Ann. Statist., 35(1):192-223. 
[5] Rivoirard, V. and Rousseau, J. (2012). Posterior concentration rates for infinite dimensional exponential families. Bayesian Analysis, 7:311-334.

[6] Rousseau, J. and Szabo, B. (2015). Asymptotic behaviour of the empirical bayes posteriors associated to maximum marginal likelihood estimator. Technical report.

[7] van der Vaart, A. W. and van Zanten, J. H. (2008). Rates of contraction of posterior distributions based on Gaussian process priors. Ann. Statist., 36(3):1435-1463.

[8] Wong, W. H. and Shen, X. (1995). Probability inequalities for likelihood ratios and convergence rates of sieves mles. Ann. Statist., 23:339-362.

CEREMAde, University Paris Dauphine Place du Maréchal deLattre de Tassigny

E-MAIL: rousseau@ceremade.dauphine.fr
LEIDEN UNIVERSiTy, Mathematical Institute, Niels Bohrweg 1, Leiden, 2333 CA, The Netherlands

E-MAIL: b.t.szabo@math.leidenuniv.nl 